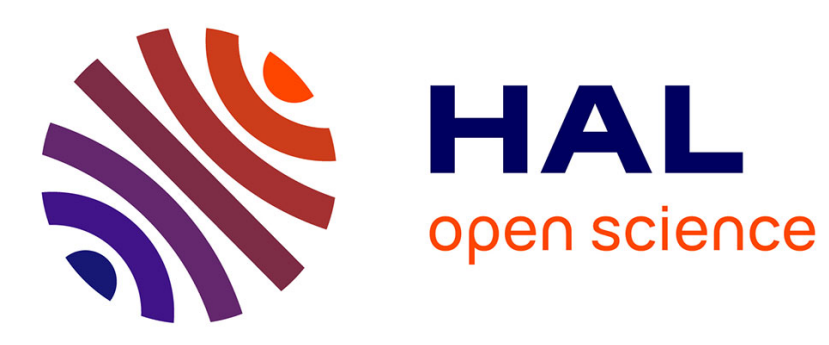

\title{
Being an Avatar "for Real": a Survey on Virtual Embodiment in Augmented Reality
}

Adélaïde Genay, Anatole Lécuyer, Martin Hachet

\section{To cite this version:}

Adélaïde Genay, Anatole Lécuyer, Martin Hachet. Being an Avatar "for Real": a Survey on Virtual Embodiment in Augmented Reality. IEEE Transactions on Visualization and Computer Graphics, 2021, pp.1 - 20. 10.1109/tvcg.2021.3099290 . hal-03320680

\section{HAL Id: hal-03320680 \\ https://hal.inria.fr/hal-03320680}

Submitted on 16 Aug 2021

HAL is a multi-disciplinary open access archive for the deposit and dissemination of scientific research documents, whether they are published or not. The documents may come from teaching and research institutions in France or abroad, or from public or private research centers.
L'archive ouverte pluridisciplinaire HAL, est destinée au dépôt et à la diffusion de documents scientifiques de niveau recherche, publiés ou non, émanant des établissements d'enseignement et de recherche français ou étrangers, des laboratoires publics ou privés. 


\title{
Being an Avatar "for Real": A Survey on Virtual Embodiment in Augmented Reality
}

\author{
Adélaïde Genay, Anatole Lécuyer, and Martin Hachet
}

\begin{abstract}
Virtual self-avatars have been increasingly used in Augmented Reality (AR) where one can see virtual content embedded into physical space. However, little is known about the perception of self-avatars in such a context. The possibility that their embodiment could be achieved in a similar way as in Virtual Reality opens the door to numerous applications in education, communication, entertainment, or the medical field. This article aims to review the literature covering the embodiment of virtual self-avatars in AR. Our goal is (i) to guide readers through the different options and challenges linked to the implementation of AR embodiment systems, (ii) to provide a better understanding of AR embodiment perception by classifying the existing knowledge, and (iii) to offer insight on future research topics and trends for AR and avatar research. To do so, we introduce a taxonomy of virtual embodiment experiences by defining a "body avatarization" continuum. The presented knowledge suggests that the sense of embodiment evolves in the same way in AR as in other settings, but this possibility has yet to be fully investigated. We suggest that, whilst it is yet to be well understood, the embodiment of avatars has a promising future in AR and conclude by discussing possible directions for research.
\end{abstract}

Index Terms-Augmented Reality, Avatar, Sense of Embodiment, Psychology, Social and Behavioral Sciences

\section{INTRODUCTION}

$\mathrm{U}$ SING our own body seems so natural: all one needs to do is intend to make a movement for it to happen. However, this ability conceals complex processes that have occupied many neuroscientists, psychologists, and philosophers, aiming to explain what causes and affects the sensation of having a body [1]. This feeling, called the sense of embodiment (SoE), is described in the research of Kilteni and Slater [2] as being composed of three sub-components: Agency (i.e. the sense of being the author of a body's movements), Body Ownership (i.e. the feeling that the body is the source of experienced sensations) and Self-Location (i.e. the sense of being located inside the body).

These senses are always present and seem to impede the dissociation of our body from ourselves. However, it was shown that the application of the right sensory input at the right time could remove this barrier and lead to the illusion of having another body. The Rubber Hand Illusion (RHI) of Botvinick and Cohen [3] is the most famous illustration of this phenomenon, producing the feeling of owning a rubber hand through synchronous visuotactile stimulation. Many other experiments followed this one by extending it to fullbody mannequins [4], and eventually to virtual avatars [5].

Defined by Bailenson and Blascovich [6] as "perceptible digital representations whose behaviors reflect those executed [...] by a specific human being", avatars provide a natural integration of users in virtual environments. The enlarged possibilities provided by Virtual Reality (VR) displays led them to be largely involved in SoE research and allowed the identification of many factors influencing the SoE. Among them, the avatar's appearance [7], interaction

- Adélaïde Genay and Martin Hachet: Inria, Bordeaux, France. E-mail: adelaide.genay@inria.fr, martin.hachet@inria.fr

- Anatole Lécuyer: Inria, Rennes, France.

E-mail: anatole.lecuyer@inria.fr

Manuscript received July 31, 2020; revised July 9, 2021. with virtual elements [8] or sensory feedback [9] were show to affect ownership illusions importantly. Bottom-up factors such as user personality traits were also found to have a play in user ability to experience embodiment (e.g. locus of control [10], sensory suggestibility [11]).

Advances in Augmented Reality (AR) have increasingly led researchers to use this technology to explore these topics too. When embodied in AR, avatars enable users to see themselves in the real world but within a body that is different in shape, size, or appearance. This possibility can be relevant in many cases, whether it be in education [12], video games [13], remote collaboration [14], medical practice [15], or simply as a means of expression [16]. Additionally, the possibility that the psychological effects of embodiment witnessed in VR may also occur in AR raises a great deal of interest. In particular, the Proteus Effect [17], which comes out as a change in user behavior and perception during embodiment, could be exploited in medical treatments and therapies [18], [19].

Along with the evolution and expansion of AR technologies, more and more research has started to address these subjects. Indeed, MR technologies can produce virtual ownership illusions similar to those seen in virtual environments by using coherent sensory feedback [20], [21]. However, still little is known about how the mix of virtual and real elements interacts with these illusions and it is not clear if they differ from the ones experienced in fully real or virtual worlds [22]. Given the growing prevalence of AR technologies and the increasing number of applications offering virtual overlays to their users, researching the embodiment mechanism in such visual environments appears to be more important than ever.

This literature review seeks to gather existing knowledge on the embodiment of virtual avatars in AR and to identify the key challenges of its implementation. To describe and categorize previous work, we introduce a taxonomy 
of virtual embodiment experiences by defining a "body avatarization" continuum. The knowledge collected here is presented in light of this continuum and grouped to draw out the features of virtual ownership illusions in mixed environments. Our contribution provides a better understanding of avatar embodiment in AR, but it also shines the light on many gray areas that have been little explored and for which we provide lines of research.

\subsection{Methodology}

This article focuses on the question of the SoE of virtual avatars in AR. It offers a review of the literature taking into account both technological and psychological aspects. The following research questions were used to guide the selection and analysis of the literature:

- What does the term "sense of embodiment" refer to in AR literature?

- Which tools and protocols are used to build an SoE in AR?

- Which measurement methods exist to evaluate the SoE in AR?

- What differences and similarities are there between the SoE in AR, VR, and reality?

- How is the SoE connected to other AR notions such as the mix of realities or the user's body visibility?

To restrict the amount of material to be covered, some concepts were discarded by choosing specific definitions of the studied notions. For example, as Kilteni and Slater [2] pointed out, the SoE is to be distinguished from embodiment itself, which changes of meaning depending on the context. Often, the term embodiment alone refers to the representation of a person in a general manner. Embodiment can also be associated with various concepts, including embodied cognition [24] or embodied interaction [25] that we will deliberately not address. Instead, we will focus on the sense of embodiment defined as a set of sensations that occur jointly with the feeling of being inside, possessing, and controlling a body [2]. Thus, this definition excludes studies such as those of Shapiro et al. [26], using virtual agents controlled by artificial intelligence. It also puts aside publications where the embodied avatar is not visible or experienced in any way by its user, as is the case for most remote collaboration tools where only the interlocutor can interact with the embodied avatar.
We comply with the definition of AR given by Azuma [27] that specifies it as a system with the following three characteristics: "1. Combines the real and virtual, 2 . Is interactive in real-time, and 3. Is registered in three dimensions". This definition puts aside avatars superimposed on the real world without any spatial coherence. Avatars viewed on screens or projections that display a virtual environment (e.g. Frohner et al. [28]) are also set aside. Lastly, avatars visualized through "mirrors" are not revoked as long as they give the impression of being the reflection of the real world (e.g. Javornik et al. [29]).

\subsection{The Body Avatarization Continuum}

AR enables virtual body augmentations and transfigurations at different levels, ranging from the simple addition of virtual body accessories to a complete change of appearance and morphology. These virtual augmentations further enlarge the already complex phenomenology of embodiment experiences. In his attempt to categorize them, de Vignemont [30] stressed the need for a taxonomy capable of capturing this diversity. We introduce a body avatarization continuum to address the manifold of virtual embodiment and to clarify what we consider "being an avatar" is. Inspired by the reality-virtuality continuum of Milgram et al. [23], we aim to describe the scale of the virtual user representation as presented in Figure 1.

At the extreme left of the continuum, the user deals only with their real body, deprived of any virtual element. This representation corresponds to the natural physical appearance of users within their daily lives. At the opposite end is what we pinned as full avatarization: the user embodies and controls a complete virtual body. This body can be visualized either in the first person through the eyes of an avatar, or in the third person as in out-of-body experiences. In the latter case, considering such experience as full avatarization may seem paradoxical, especially when the user's real body remains visible. However, previous research suggests that the SoE can occur towards disconnected virtual avatars as well [31], [32], [33]. We, therefore, have included them in this area of the continuum.

The region between the two extrema of the continuum relates to diverse degrees of virtual body augmentation. In this area, we distinguish body accessorization from partial avatarization. Body accessorization describes the addition of superficial elements such as virtual clothes or glasses to the user's body. Examples of such augmentations can be found

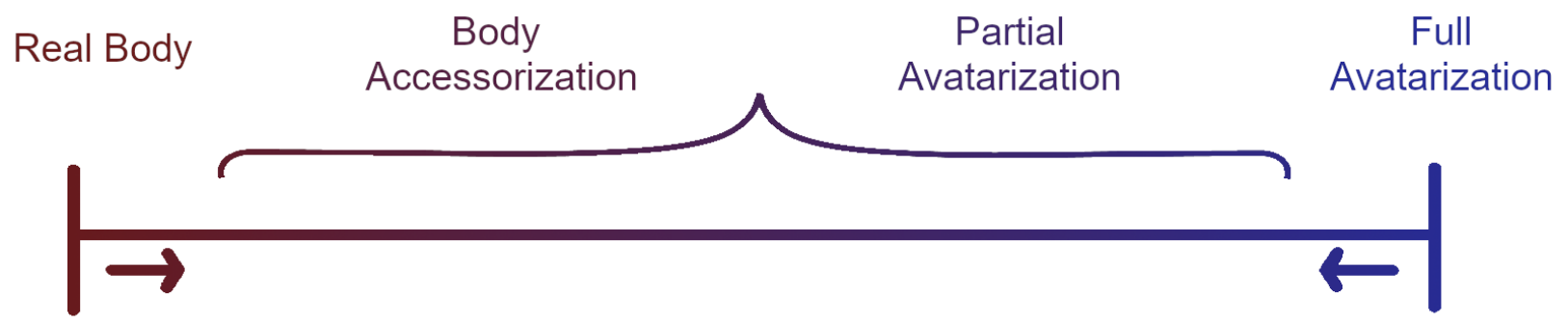

\section{Body Avatarization Continuum}

Fig. 1. The body avatarization continuum covers the extent of the user's virtual representation within a virtual embodiment system, regardless of the environmental context. It is inspired by the Reality-Virtuality Continuum proposed by Milgram et al. [23] on the virtuality degree of an environment. 
in the projector-camera system of Harrison et al. [34] where a watch is displayed on the user's wrist.

Partial avatarization involves the addition of virtual body parts or removal of real ones as well as the modification of bodily characteristics. This includes limbs, skin color, face shape, and other features integrated into the human anatomy. For instance, the supernumerary hand illusion implemented by Gilbers and Rosa [1] corresponds to partial avatarization as it provides the user with an additional limb - a third hand - integrated into their real body. Whether the modification of traits such as hair length, eye color, etc. corresponds to partial avatarization or body accessorization is up for discussion. Being customarily modified in real life (e.g. through hair extensions, contact lenses), we decided to place such changes between these two areas.

The question of where body augmentation starts being avatarization is another complex subject, falling within the realm of epistemology [30]. On the one hand, removing or placing accessories on our body will not change the philosophical notion of "me" [35]. On the other hand, when choosing attire, we assume a particular social identity that elicits corresponding behaviors linked to our image of society [36], [37]. Indeed, it seems that clothes and accessories do not only impact the way our entourage perceives and judges us, but also the way we see ourselves. A significant number of studies seem to indicate that this self-image has quite important consequences on our behavior, often in unconscious ways [38]. For instance, people wearing formal clothes might want to convey the image of a professional and serious person, but will also feel more focused and negotiate with more confidence when wearing such clothes [39], [40]. This top-down influence named "enclothed cognition" (Adam and Galinsky [37]) is reminiscent of the embodiment of avatars: our intrinsic identity remains the same, but wearing clothes and accessories broadcasts a selfrepresentation that we control and identify to. As an effort to resolve ambiguity, this article assumes that virtual body accessorization eventually amounts to embody a hybrid avatar, i.e. a representation mixing real and virtual elements.

In the following sections, the term "body avatarization" will be used indistinctly to refer to the implementation of any of the virtual embodiment experiences defined by the continuum.

\section{Body Avatarization in the AR Literature}

Body avatarization in AR can be useful in a large variety of applications. As it is impossible for us to make an exhaustive list of them, this section presents the use cases that were the most investigated in the AR literature for each part of the body avatarization continuum.

\subsection{Body Accessorization}

Straightforwardly, one of the primary use cases for body accessorization in AR resides in retail. By overlaying products on the user's body, this type of augmentation can not only improve consumer experience (both in-store and online) but also remove potential discrepancies between products and perceived body sizes [41]. Examples of AR try-on systems letting shoppers virtually fit clothes, jewelry, eyewear, or shoes are abundant in both academia and industry [34], [42], [43], [44], [45]. More details on their experiential value can be found in existing surveys on that subject [46], [47].

Body accessorization has also been used in entertainment, artistic and cultural activities. Its combination with AR provides users with a novel way to convey ideas by letting them change their attire dynamically. Among examples, the virtual make-up application MagicFace [29] was deployed in a museum and in an opera house to let visitors observe how they would look with Egyptian pharaoh makeup. Its creators see a broader use for such applications: they could help make-up artists in their creation process or aid on-stage performers step into character without having to dress up. These ideas were explored by Bermano et al. [48] who proposed a live facial make-up system designed for novel performances. Treepong et al. [49] also implemented a tool aiming to improve make-up creativity. In similar lines of work, AR dynamic dresses were proposed by Kleinberger and Panjwani [44] as a "new form of expression through clothing to reflect identity, personality, emotions and inner states of the wearer".

\subsection{Partial Avatarization}

Partial avatarization finds great relevance in the medical field. For instance, severed patients could use it to train themselves to use a virtual prosthesis before implanting a real one [50]. Research has shown that this could not only help to accelerate user adaptation but also reduce phantom limb pain and lower rejection rates through the development of long-lasting ownership illusions [51], [52]. Similarly, partial avatarization in AR could help to recover motor functions during rehabilitation after a stroke [53], as done by Heinrich et al. [54]. Indeed, virtual embodiment illusions applied to paralyzed limbs were found to ease recovery by generating cognitive stimulation to the brain linked to body ownership, agency, and kinesthetic perception through visual feedback [55], [56].

Instead of replacing or overlaying a human limb with an equivalent virtual one, partial avatarization can also be used to modify one's body structure and extend our possibilities of interaction. Feuchtner and Muller [57], for example, implemented an AR system replacing the user's real arm with an expandable virtual one, allowing to reach objects over twice as far without breaking the user's sense of ownership (Figure 3). They further demonstrate the feasibility of using virtual arms to interact with real objects connected to the system (e.g. actuated table, electric curtains). Lastly, Wang et al [58] showed that AR virtual hands could help people with hand tremors to type more efficiently. Their system tracked the user's hands and overlaid stabilized hand models over them in a slightly transparent way for the users to see the keyboard underneath. Their study illustrates how AR partial avatarization can be used to facilitate daily tasks for physically impaired or disabled people.

\subsection{Full Avatarization}

Full avatarization in AR extends what can be done with previous parts of the continuum: one could use it to embody an avatar with a different appearance, shape, and size, or to observe oneself in a given (real) situation through 
virtual out-of-body experiences. Examples of papers using full avatarization in the AR literature are extremely rare. Most of research implementing such avatarization were user studies on perception and not application-oriented papers [21], [31], [32], [59].

Among the rare examples of application papers we found, the study of Anderegg et al. [60] is an example of how AR full-bodied avatars offer compelling possibilities of original game-plays. They were interested in novel ways to control full-bodied avatars in AR mobile games. The interaction metaphor they propose lets players control their avatar like a puppet with intuitive gestures. Hoang et al. [61] and Johnson and Sun [12] illustrated how full avatarization can also be used in educational and health awareness settings. Both of their studies proposed SAR full avatarization systems exploring on-person anatomical displays for health education (muscles, blood vessels, etc.). Their results demonstrated a stronger level of "connectedness" and ownership with the projected content than with other projection supports, especially when the data was coherent with the participant's physiological state (e.g. synchronized heartbeat).

The lack of other examples in the literature is probably due to the difficulty of implementing full avatarization in AR (see Section 3.2 for further details). Nevertheless, one can imagine a large number of scenarios where full-bodied self-avatars could be useful, including in therapy, training or self-expression. We discuss these potential use cases in Section 7.3.

\section{Implementing Body AVATARIZATION IN AR}

While the release of multiple frameworks (e.g. ARFoundation ${ }^{1}$, Windows MRTK $^{2}$ ) has considerably simplified the development of AR applications, setting up a sense of embodiment remains a truly complex task in AR. The

1. Unity (2020) https://unity.com/fr/unity/features/arfoundation

2. Microsoft Corporation (2020) https://github.com/microsoft /MixedRealityToolkit-Unity purpose of this section is to constitute a guide to avatar embodiment in AR. We describe the core components of AR avatarization and break down the existing possibilities to implement strong ownership illusions in AR. Examples of applications will be given to illustrate the current uses of each modus operandi.

\subsection{The Choice of User Perspective}

To make body avatarization and ownership illusions possible, technical choices are to be made conscientiously as they could lead to very different user experiences. The first of these important decisions is from which perspective the avatar should be embodied.

In a first-person perspective (1PP), the user will look through the eyes of their avatar and/or experience embodiment by looking down at their own body (e.g. Figure 2, left). This point of view can be combined with virtual mirrors that let users observe their body avatarization indirectly (e.g. Figure 2, center). In a third-person perspective (3PP), the avatar will be observed at an arbitrary location and result in an out-of-body experience (e.g. Figure 2, right). For example, instead of seeing a virtual arm where one's real arm would naturally be, one could see it floating ahead and use it to grab normally unreachable objects thanks to partial avatarization.

The choice for either perspective has to be made in consideration of two factors. The first is the application's purpose: does the user need to see specific parts of their body, like their face? If so, an egocentric point of view will only let users see virtual content at locations that they can directly see. Augmentations positioned on faces, necks, or backs will not be visible in this point of view and therefore limit certain avatarization applications unless combined with virtual mirrors. In the third-person perspective, users can observe their virtual avatarization in its entirety from different angles and standing viewpoints, providing them with an external awareness of their avatar.

The second factor to take into account is the strength of the ownership illusion that is sought. We are not aware
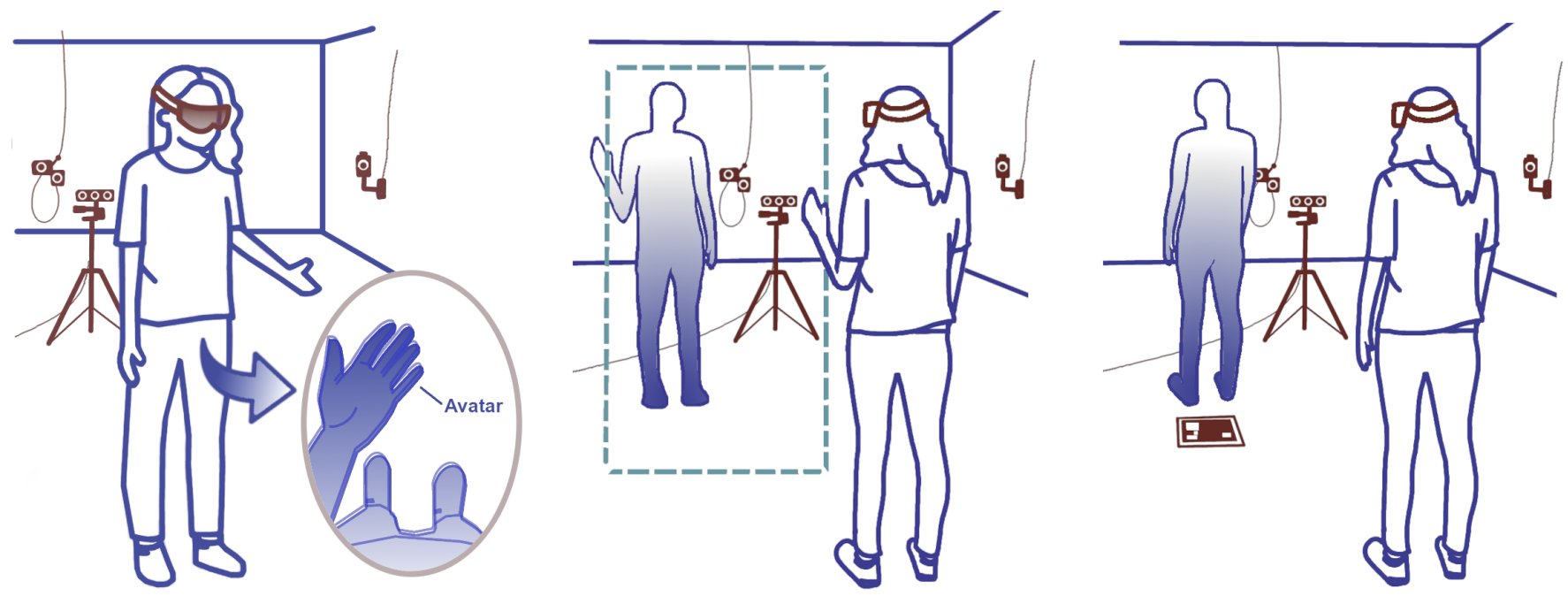

Fig. 2. First and third-person perspective in an AR full avatarization setup. Left: The user wears an AR HMD and looks down onto her fully avatarized body. Center: A virtual mirror reflects the user's body. Right: The user observes her self-avatar instantiated ahead of a fiducial marker with a body pose identical to hers. 
of studies that have compared 1PP and 3PP in AR. Nevertheless, the first-person viewpoint preserves the instinctive perception of self-location with respect to the real body and this seems essential to generate a strong SoE. Some VR studies support that 3PP can generate equal ownership illusions as 1PP [62], but the major part of the community seems to agree that egocentric points of view will produce a stronger sense of ownership and self-location [63], [64], [65], [66], [67]. This being said, 3PP can provide illusions strong enough to satisfy some AR embodiment applications [31], [32]. This perspective also has the advantage of increasing awareness of the virtual posture and of its surroundings [62] and therefore justifies choosing it over 1PP in some cases.

Ultimately, we would recommend applications that seek maximum ownership illusions to use $1 \mathrm{PP}$ and to combine it with virtual mirrors when applicable. However, as of today, examples of full avatarization in egocentric perspectives like the one in Figure 2 (left) are still rare in AR; most of the existing 1PP applications content themselves with partial avatarization by solely displaying virtual hands [68]. In fact, one main problem with this embodiment point of view is that tracking errors are very likely to happen, even with state-of-the-art technology, and that the consequent shifts between the real and virtual bodies are more prone to be noticed. When too pronounced, these inaccuracies can impair the usability of the avatar, and as a result, the embodiment experience too [69], [70]. Section 3.2 describes some solutions for this issue in accordance with the type of display used.

\subsection{Displaying Avatars in AR}

Avatar embodiment can be experienced in AR through a diversity of displays with different modes of operation (e.g. head-mounted, handheld) and different renderings (e.g. video, optical). In this section, we will not detail how these displays work or the advantage and issues that are specific to them (see [71], [72] for a complete overview). Instead, we will discuss how their characteristics make them more prevalent or less suited for embodiment experiences located at different parts of the body avatarization continuum.

Non-immersive Video See-Through (VST) displays: they are monitor-based systems also known as "windowson-the-world" (WoW) displays. Smartphones and tablets are common examples, but they could also be larger screens (e.g. Figure 4, right). When implementing body avatarization, their main strength is that they can be combined with live image processing to erase the user's real body through in-painting techniques [71]. This allows the partial and full avatarization of bodies with a morphology different from the user's that would otherwise be impossible (e.g. 1PP partial avatarization of a thinner arm). However, the screen sizes of WoW displays are often limited and will usually not let users see the whole virtual content at once. Most of the user's body consequently remains visible outside of the display during 1PP full-body avatarization. In previous work, such displays were mostly used to implement virtual mirrors [29], [42], [49], [68].

VST Head-Mounted Displays (HMD): this mostly refers to VR headsets that are equipped with external cameras (e.g. Oculus Quest 2, HTC Vive Pro). They seem better adapted to immersive 1PP embodiment experiences than non-immersive VST displays as they completely hide the direct vision of the user's real body. Combined with inpainting, 1PP full avatarization can be achieved at its best by completely overriding the real body's vision with the one of a full avatar, regardless of its morphology as done in Figure 3.

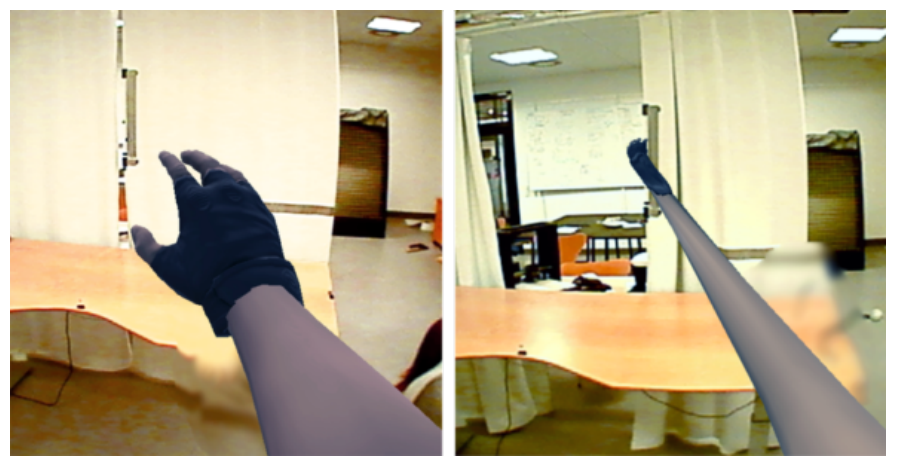

Fig. 3. Example of partial avatarization with a head-mounted VST display (Feuchtner and Müller [57]). Left: A virtual arm of normal length is embodied in first-person AR. Right: The user extends his virtual arm towards a normally unreachable (real) electric curtain and manipulates it.

Optical See-Through (OST) HMDs: unlike their VST counterpart, OST headsets provide an unmediated view of the real world (e.g. Microsoft Hololens ${ }^{3}$, Magic Leap ${ }^{4}$ ). On the one hand, this direct view "ensures that visual and proprioception information is synchronized" as described by Rolland and Fuchs [73]. On the other hand, virtual content viewed with them is partly transparent and sometimes causes depth perception issues that may impact the embodiment experience, as witnessed by Gilbers et al. [1]. Another issue with these headsets is that their usually narrow fields of view can break the continuity of ownership illusions as parts of the avatar might get cropped by the optical system and let the user see their real body in the outer areas of the display.

Projection-based systems: this refers to Spatial Augmented Reality (SAR) and CAVE-like systems. Like in OST, the opacity of content rendered with SAR heavily depends on light output capacity and ambient lighting. Using it for partial and full avatarization is more complicated when the embodied limb or avatar is not matching the user's body or projection surface shape. Stereoscopic CAVE systems provide more latitude, but removing the visibility of real user body parts presents the same problems and may require ingenuity (e.g. Slater et al. [74]). Despite their restrictions, projection-based systems can nevertheless suit the needs of particular embodiment applications [12], [75]. For instance, Johnson and Sun [12] used SAR to explore 1PP partial avatarization by allowing users to interact with their own internal makeup projected on their body (i.e. muscle tissues, blood vessels, etc.). SAR was also previously used for 3PP embodiment of differently shaped bodies: Gervais et al. [76], for example, designed a small plastic figurine representing the user's inner state as a means of introspection. When em-

3. Microsoft (2020) https://www.microsoft.com/

4. Magic Leap, Inc. (2020) https://www.magicleap.com/ 
bodying it, user physiological data is recorded and projected onto it.

OST "fishtank" setups: these systems use semitransparent mirrors to reflect AR content displayed on a stereoscopic screen [22], [58], [77], [78], [79]. They can be used to overlay 3D content on and around the user inside of a dedicated tracked volume under the display's mirror. In general, this volume is small and will only allow body accessorization and partial avatarization (e.g. Shibuya et al. [79]). OST fishtanks present the same drawbacks as projection-based systems.

Overall, current trends seem to favor HMDs as the primary medium for AR display. HMDs have the advantage to combine freehand interaction with multiple built-in sensors that provide realistic integration of virtual content [80]. Ultimately, however, there is no one-size-fits-all solution for all embodiment applications. Each trade-off must be evaluated in light of particular goals and available technologies to decide which form of display is best.

\subsection{Controlling and Animating Avatars in AR}

A component that is essential to virtual avatarization is the control of the embodied avatar. While it is possible to achieve an SoE without any avatar animation [3], giving control of it will enable interaction and strengthen body ownership illusions [81] (see Section 5.1). Virtual avatars can be animated by users in many ways, including with tangible interactions [82], controllers [83], puppeteering [60], [84] or motion tracking technologies. These methods are not specific to $A R$ and their usage for body avatarization is the same as in VR applications. However, they come with their own challenges when used in real-world contexts for embodiment.

To our knowledge, no study explored the influence of different animation techniques on the SoE. However, the sense of agency is known to be greatly enhanced by visuomotor congruence between real and virtual body movements, while inconsistencies between visual and motor information appear to reduce it [85], [86]. Control through inputs that are not physically representative of the user's body (e.g. with gamepads) might therefore not play in favor of strong ownership illusions. For this reason, tracking technologies that provides a 1-to-1 mapping of user movements has been the preferred tool for user control during embodiment [87].

Numerous motion tracking technologies exist, ranging from open-source all-in-one software programs to costly hardware-based solutions (see Spanlang et al. [87] for details). Whereas reflective marker-based systems are the most widespread in VR research, optical markerless tracking seems equally common in AR avatar embodiment applications [29], [31], [32], [42]. Markerless tracking consists of recording the position and rotation of the user's body key points in real-time. Free toolkits and libraries can be used to record these points (e.g. OpenPose [88], Unity's AR Foundation ${ }^{5}$ ). Once collected, they are matched to the avatar's corresponding body or face landmarks and the 3D model is animated into the same pose or expression as the user (Figure 4). For the full-body posture to be deduced out

5. Unity Technologies (2020) https:/ / unity.com/ of this data, Inverse Kinematics (IK) solvers are commonly used: they calculate joint angles based on body constraints so that only natural poses will be taken by the avatar [89].
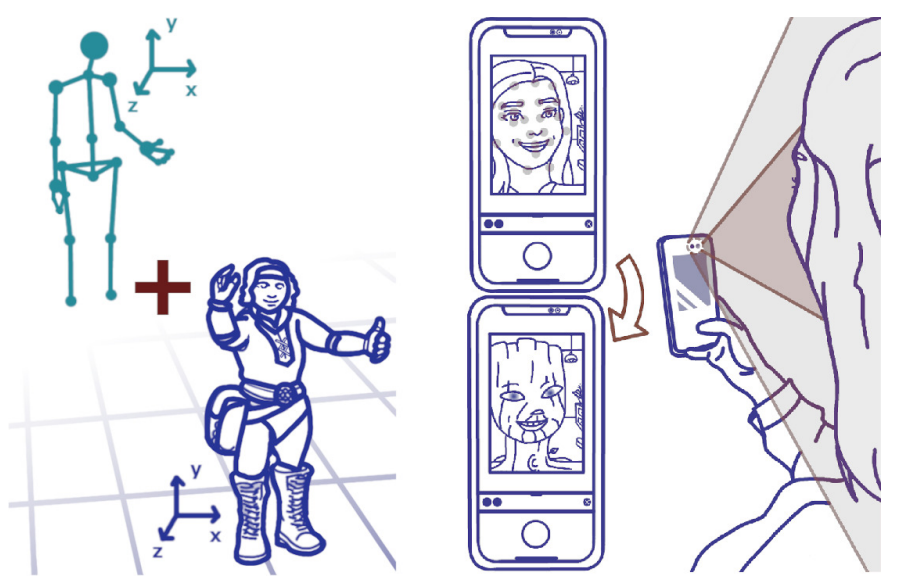

Fig. 4. Animation process in AR. Left: During full avatarization, a skeletal pose is acquired and matched to animate the avatar into the optimal estimated pose. Right: Face feature points are extracted to morph the avatar model for partial avatarization of the user's face. Here, it is visualized on a smartphone acting as a virtual mirror.

All of these processes make tracking and image generation inevitable sources of delay in AR systems, even with high refresh rates [90]. The effects of tracking errors on the SoE were not clarified in AR, but VR studies have found that certain forms of errors including latency, jitter, and noise can strongly affect the SoE [91]. According to Toothman and Neff [70], latency and noise (avatar vibration) appear to be particularly limiting the SoE at relatively low thresholds.

Noise and jitter are errors linked to the tracking signal which quality may vary importantly with the real environment's conditions (e.g. lighting). Providing robustness for these errors is still a hot topic of current research [70], [92]. One solution to counter the latency issues of AR systems is to use VST displays. Indeed, a video view of the real world makes it possible to synchronize the overlay of virtual and real content by deliberately delaying video images to match the tracking and rendering latency [93]. On the other hand, the real world view of projection-based and OST systems offers no latency and therefore makes it impossible to completely compensate entirely for delayed tracking: one can reduce misalignment between synthetic and real imagery through predictive tracking and selective update, but errors are still likely to be present, especially during rapid head pose changes [94].

To conclude, motion-based tracking promises stronger illusions than its counterparts, but it comes with its share of technical difficulties in AR. Depending on the type of display used, it will introduce errors that can disrupt embodiment illusions and that may require effort to be handled. Latency is the most prominent source of registration error in existing AR systems [95]. Because of the need to maintain alignment between real and virtual environments, avatarization applications in AR have to make an extra effort to preserve the user's sense of agency compared to VR. 


\subsection{Integrating Avatars to the Real World}

Ultimately, it seems that AR embodiment systems rely on similar components as the core VR embodiment system depicted by Spanlang et al. [87]. The main difference they present resides in the type of displays used and in the replacement of the VR module by an AR one. However, this AR module has to accomplish an additional task that makes AR embodiment much more complex: it has to build spatial awareness of the physical environment to handle the proper integration of virtual content.

Several techniques exist to produce an accurate 3D registration of virtual avatarization in real space. The detection of fiducial makers, planar images, or 3D objects is a long-dating and simple way to position avatars at the desired location [96], whether it be on the user's body [15] or elsewhere [32]. This method involves tracking markers registered beforehand in the AR system. Once the marker is recognized, the avatar is spawned in a location relative to it and displayed (e.g. ahead like in Figure 2, right). Such process is fast to set up thanks to the availability of many free toolkits ( $\mathrm{ArUco}^{6}, \mathrm{ARToolKit}^{7}$, etc.), but alone, it does not provide the embodiment system with any understanding of the physical environment. Without such understanding, the relative position of real and virtual objects is ignored and the coherence of the scene cannot be ensured.

To provide occlusion between real and virtual objects, spatial awareness can be roughly acquired through Simultaneous Localization And Mapping (SLAM) algorithms [99], [100]. These techniques create an approximate model of the environment by using sensor data (mostly camera feed) to detect feature points, associate them with 3D space coordinates, and update them continuously. Systems using SLAM do not require markers as the created model can be used to identify environment or body surfaces and to place content onto them (e.g. Figure 5). Once detected, surfaces can also be virtually registered to stop virtual content from unnaturally entering walls and to implement virtual occlusion, resulting in a stronger coherence of the embodiment illusion. On the downside, they require more computing power and tend to be less stable than marker-based systems as the feature points are constructed from sensor data that is updated continuously and that may contain errors.

Examples of software capable of rendering AR content with spatial awareness are game engines (e.g. Unity, Unreal Engine ${ }^{8}$ ). They are particularly fit for such tasks as they integrate avatar animation, state-of-the-art graphics, stereoscopic rendering, realistic physics, sound, memory management, and many AR development tools. Additionally, they support a fair number of commercialized tracking systems and allow cross-platform builds of AR scenes for most mainstream AR devices.

Finally, to draw a parallel with the core VR embodiment system described by Spanlang et al. [87], we could define a core AR embodiment system composed of the following modules:

6. Garrido-Jurado et al. [97], Romero-Ramirez et al. [98] https://www.uco.es/investiga/grupos/ava/node/26

7. ARToolworks (2020) https://www.artoolworks.com/

8. Epic Games, Inc. (2020) https://www.unrealengine.com/
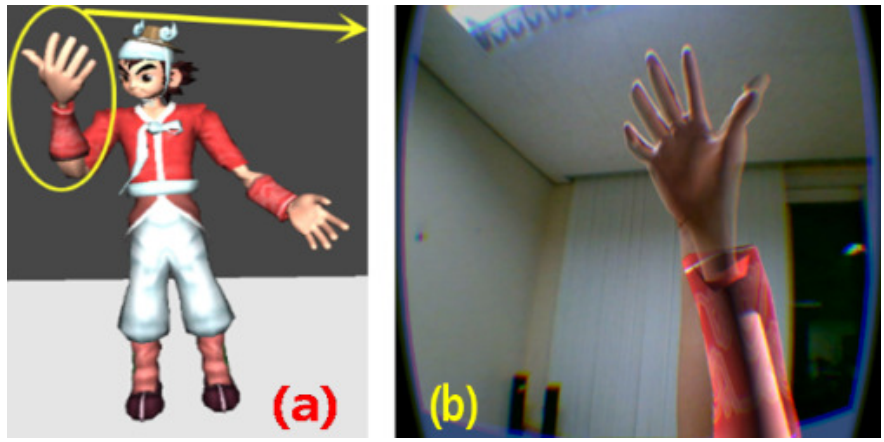

Fig. 5. Example of full avatarization in first person perspective with a VST HMD. The avatar is overlaid on the user's real body which is rendered partly transparent. Extracted from the paper of Noh et al. [14].

- Tracking Module: depending on the type of display (i.e. handheld vs. head-mounted) records the user's body and head movements or display device's position in real-time. It allows controlling the avatar and adapting the AR scene point of view. This tracking can be done with the technologies referred to in 3.3 and is used as an input of the AR module.

- AR Module: processes the outputs of every other module and adapts the rendering of the AR scene according to them; takes in charge the integration of the avatar into the real world from the knowledge acquired through the tracking module or separate environment processing (e.g. SLAM); passes the resulting rendering to the display module.

- Display Module: hardware allowing the user to visualize their virtual avatar and the AR scene (see Section 3.2).

The AR module is also responsible for the creation and management of the AR content and usually handles the animation and interactions related to the avatar (i.e. pose estimation, IK, etc). In more advanced systems, it may also include lighting simulation, stereoscopic rendering, and audio I/O management. Additional modules can be added to this core system in order to provide multi-modal stimulation (e.g. haptic feedback) and SoE measurement tools (see Sections 4 and 5). The output of such modules would then be passed as inputs of the AR Module so to take them into account. Finally, alternative user inputs through controllers or voice can also be combined with the user tracking system to provide different embodiment interactive experiences.

\subsection{Summary of Technical Challenges}

A variety of obstacles in adopting AR embodiment were mentioned in the previous sections. Most of them are linked to consistency issues of AR experiences [101]. Dealing with these challenges includes:

- Providing correct registration and alignment of the virtual body with the real one during $1 \mathrm{PP}$ embodiment.

- Compensating for latency causing discrepancies between the user and the avatarization movements.

- Dealing with real environmental conditions that can impact the avatarization's visibility (e.g. lighting when using OST displays). 
- Removing visible user body parts when implementing a virtual embodiment of differently structured bodies (e.g. thinner limbs).

- Ensuring that the virtual avatar responds realistically enough to the physical world (e.g. collide with walls, displays shadows).

- Providing a field of view sufficiently large to enable witnessing the avatarization as intended.

Dealing with these challenges can be more demanding or not apply depending on the targeted experience (e.g. simulated autoscopy doesn't require removing the real body). Achieving further realistic experiences of avatarization will require solving additional challenges such as letting users interact with the real world objects in addition to virtual objects, rendering in high quality, or providing haptic feedback during embodiment. In addition to these, previous literature outlined other challenges linked to the general usage of AR, including visual fatigue, security issues, marker tracking reliability, and device costs [102], [103].

However, we feel that these issues are relatively minor and should not prevent the adoption of AR for virtual embodiment. Most embodiment systems described before can already be implemented with state-of-the-art technology. Although some configurations are more challenging to achieve (e.g. 1PP full avatarization), we believe current technological limitations are unlikely to be prohibitive for most AR avatarization experiences (e.g. partial, accessorization, 3PP embodiment). New advances are anticipated to overcome current usability and technological issues such as low resolution, limited fields of view, cost, and low computing power. Lastly, the rapid growth of AR research and industry encourages the idea that this technology will soon reach the same level of usability as VR in the coming years [103].

\section{Measuring the Sense of Embodiment in AR}

As of today, measuring a psychological phenomenon as complex as the SoE in AR remains an open debate. Very few publications share the same methods and choosing the most appropriate one is not always a simple task. The tools used in AR to measure the SoE are mostly derived from those previously used in VR, which themselves have evolved greatly over time. In this section, we detail the candidate methods that could be used to evaluate the SoE in AR.

\subsection{Subjective Measures}

In the original RHI experiment, Botvinick and Cohen [3] chose to design a two-part questionnaire to assess the SoE. The first part asks for an open-ended description of participants' feelings, while the second part consists of nine questions to be answered on a Likert scale [104]. Their conclusions gave rise to a great deal of research work but were faced with a lack of validation [105], [106]. As a result, some researchers modified the questionnaire while others deleted the descriptive part or kept the descriptive part only. This made the outcomes of studies particularly difficult to compare, and therefore hard to validate.
Confronted with this problem, researchers made efforts to verify and unify measurement questionnaires through systematic analysis. Longo et al. [107] concluded from their observations that Likert scales are valid tools since it is possible to identify distinctive patterns in the answers of these questionnaires. However, they should, as much as possible, be supported by an objective measure that provides a complementary validation. In their view, the use of traditional psychological methods alone - such as open verbal description - is too subjective to extract an accurate measure of the SoE. Subjective embodiment measurement is indeed difficult because our relationship with our own bodies is not something we usually consider. Many people who have never witnessed or heard of autoscopic experiences may find questions like "I felt out of my body" unrelatable. Slater raised similar issues regarding the measurement of presence in VR with questionnaires [108].

With more hindsight, research looked to establish a standard questionnaire for better comparability while accounting for these challenges. Roth et al. [106] identified a set of fundamental questions by principal component analysis of the literature and produced a first questionnaire, then revised to contain 12 items in 2020 [109]. The questionnaire is available in 7 languages at virtualembodimentscale.com. In the meantime, Gonzalez-Franco and Peck [105] proposed their own version built on exploratory factor analysis. It was also updated in 2021 to keep a total of 16 items applicable to all scenarios, including those of AR [110]. Both questionnaires were tested and validated by several studies ( 4 and 9 , respectively) and then by statistical reliability analyses (calculation of Cronbach's alpha coefficient).

The generic nature of these two questionnaires makes them appropriate for the evaluation of AR avatarization anywhere on the continuum. Additionally, their extended testing makes them good candidates to become the new standard of SoE evaluation. However, their construction and trial were mostly based on RHI and VR studies. It would be interesting to make a comparative study evaluating their efficiency in various contexts (including AR). Indeed, it is still unclear whether mixing virtual and real content has an impact on ownership illusions. Škola and Liarokapis [20] reported no difference in their measures of the RHI in AR, VR, and reality, but it is unclear whether their results apply to avatarization of larger extents or illusions including visuomotor feedback. Regardless, using one of these two validated questionnaires in AR will enable future comparison with experiments in VR and real contexts. We therefore encourage to use them in future SoE research in AR, but also to implement control conditions to empirically test for potential bias of AR environments.

\subsection{Objective Measures}

Most objective measurements used in VR can also be applied in AR. One that is commonly found in both is that of proprioceptive drifts, characterized by the change of a perceived body or limb's location towards a fake embodied one Botvinick and Cohen [3]. This measure can be obtained by subtracting the user's estimated body or limb position from its actual position [1], [20]. However, Rohde et al. [111] and Holle et al. [112] highlighted the risks of using 
this measure alone and the necessity of combining it with complementary ones as they showed that proprioceptive drifts could also occur in the absence of SoE.

Bodily signals can be used in AR as indicators of efficient virtual hand and body ownership illusions too: when the avatar is placed under a virtual threat (e.g. a sharp knife suddenly falls), studies have shown that participants will attempt to avoid virtual danger in the case where the illusion is successful. This response to danger is reflected in several bodily signals such as the skin conductance response (SCR) and heart rate deceleration (HRD), which can be recorded in both VR and AR [64], [113]. Body ownership illusions have also been correlated with decreases of user body temperature [114] and variations of muscle electrical activity (EMG) [115]. A complete compendium of methods and technologies allowing to obtain them can be found in the paper of Spanlang et al. [87].

Lastly, brain activity can provide information on the neural processes involved. This activity can be analyzed through electroencephalography (EEG) recordings, as done in the right part of Figure 6 where Škola and Liarokapis [20] look for increased activity in EEG bands to detect effects of body ownership illusions. Some have used EEG to measure intentional binding, an internal process that was identified as crucial for the normal experience of agency [116], [117]. However, the relationship between EEG and intentional binding is still controversial [118] and EEG recording has several drawbacks when integrated into avatar embodiment applications: on top of being time-consuming to set up, most non-intrusive EEG systems are very sensitive to muscle activity and electrical noise in the environment, limiting the embodiment to an immobile experience with very little possibilities of interaction. Further details on EEG setup and signal processing for the study of body ownership illusions can also be found in the article of Spanlang et al. [87].
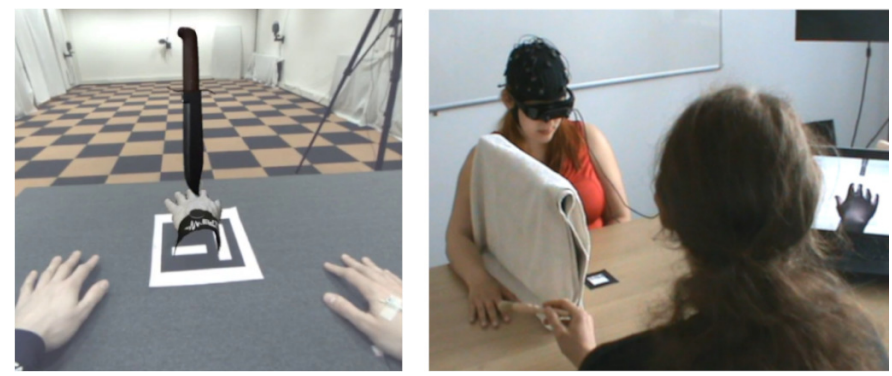

Fig. 6. Examples of objective measurements of virtual hand illusions (VHI). Left: Sensors are placed on the participant's right hand to record their SCR at the moment a virtual knife falls on the embodied hand [113]. Right: EEG recording during VT stimulation in a VHI in AR [20].

While partial and full avatarization can be evaluated straightforwardly with any of these methods, the measurement of AR body accessorization experiences raises several questions: how does it relate in any way to embodiment experiences, and how can one quantify it? According to the literature review of Schettler et al. [119], objects can be experienced as being "a part or extension of one's body". To support this idea, they relate an experiment where researchers were able to observe and measure the effects of ownership illusions of a table [120]. The illusion was induced by stroking synchronously a table and the participant's hand after having applied a Band-Aid on both of them. Embodiment effects were measured with a questionnaire and SCR that showed that participants somehow felt that the table was their hand. Indeed, the questionnaire answers were corroborated by a peak in SCR upon partly pulling off the table's Band-Aid, leading user to expect pain. Neuropsychological research further supports the idea that inanimate objects can influence the body image and schema: Aglioti et al. [121] described a case where a patient with a brain lesion had no sense of ownership over their arm but could recognize it again after removing their wedding ring. These results suggest that body accessorization (whether in AR or not) does have an influence on body ownership and that this influence should be measurable with the same methods as in other avatarization experiences.

\section{Inducing the Sense of Embodiment in AR}

Generally, the strategy used by researchers to induce the SoE in AR experiments is the same as in VR or in reality: it consists of creating and intensifying the agency and body ownership senses towards the avatar. In this section, we describe the methods classically used in literature to induce and maximize these senses in AR. The methods described here apply to body avatarizations from all parts of the continuum.

\subsection{Visuotactile Stimulation in AR}

At the very beginning of experimental research on the SoE, researchers placed themselves in a simpler, entirely real setting. First, Botvinick and Cohen [3] used synchronous visuotactile (VT) stimulation to create the illusion of owning a rubber hand (i.e. the RHI). The experiment consisted of stroking a fake hand with a brush simultaneously with the participant's real hand, hidden from sight. Petkova and Ehrsson [4] generalized this experiment to the whole body in the development of a full-body ownership illusion by giving the point of view of a plastic human mannequin to participants. While touching both bodies identically, they managed to create the illusion of embodying the mannequin. The same question then arose in the context of VR and AR: can VT stimulation make one feel embodiment towards a 3D avatar? If so, how can this feeling be maximized?

To begin exploring this subject, Raz et al. [122] designed a variant of the RHI called the Virtual Hand Illusion (VHI). This variant implements partial avatarization by replacing the rubber hand with a 3D virtual hand. It applies the same synchronous VT stimuli as in the RHI. Whether in AR or VR, the comparison made by Škola and Liarokapis [20] showed that VT stimulation could evoke an SoE towards a virtual hand very close to the one evoked by a physical copy of this hand.

The VHI experiment was also generalized to the whole body to see whether VT stimulation could generate fullbody ownership illusions during full avatarization too. As shown by several literature reviews on that subject, scientists mostly turned to VR to set up this variation [5], [123]. A large number of implementations were developed using this technology showing multiple times the effectiveness of VT stimulation with several types of avatars [5]. We could not 
find examples of full body ownership illusions of avatars with VT stimulation in AR research, but it is likely that similar results would be obtained.

\subsection{Visuomotor Stimulation in AR}

Like VT stimulation, visuomotor (VM) stimulation can be used to induce embodiment illusions [124], [125], [126]. Examples of VHI and full-body ownership experiments demonstrating its efficiency can be found in the publications of Suzuki et al. [127], Nimchareon et al [59] or Genay et al. [22]. In these variants, the tactile stimulus was replaced by the ability to move the virtual body. As with VT stimulus, synchronization of real and virtual movements was shown necessary to create embodiment illusions [1].

VM stimulation is by far the most implemented stimulus to induce an SoE in AR embodiment studies and applications [21], [22], [31], [32], [79]. Compared to VT stimulation, VM stimulation is generally cheaper, easier to setup and easier to automatize (see Section 3.3). Automated VT stimulus in AR requires integrating haptic devices that can be more constraining or costly, especially when the user ought to physically move around and interact [128].

One important issue that remains uninvestigated is the suppression of visual stimulus in AR. The experiment of Slater et al. [64] showed that it is possible to exhibit a strong SoE in VR without having to stare at the embodied avatar, hence reflecting the results of the Somatic Hand Illusion of Ehrsson et al. [129]. The authors explain that the sense of agency coupled with the first-person vision of a virtual world rich in realistic detail had led to proprioception strong enough to suppress the need to look at one's body to feel like embodying it. Thus, the theoretical question of whether the absence of a direct view on the avatar can provide identical results in AR can be raised.

\section{IMPACTING FACTORS OF AR EMBODIMENT}

VR research identified many factors of influence of body ownership illusions including avatar appearance, embodiment point of view, sensory feedback, personality, environment, and avatar control [9]. It is not clear whether body avatarization in AR is impacted by the same factors, and
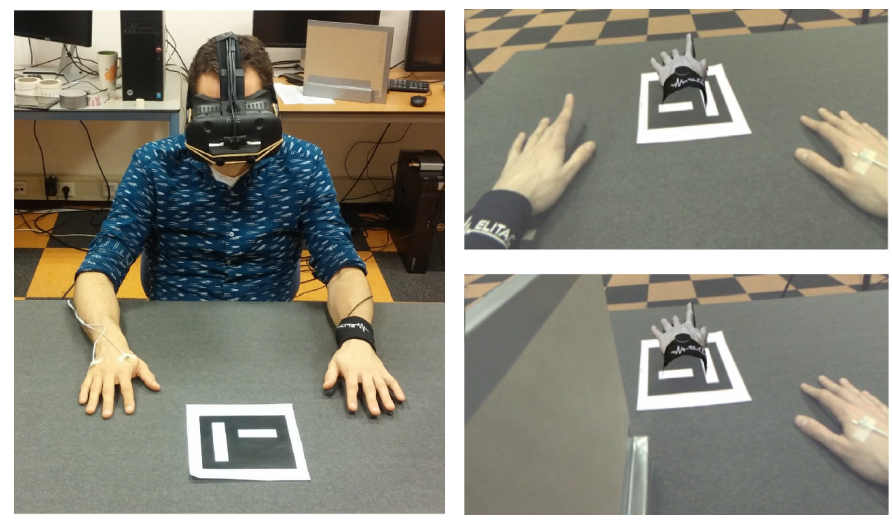

Fig. 7. Virtual hand illusion (bottom right) and supernumerary hand illusion (top right) implemented in AR by Rosa et al. [113]. Left: The experimenter's point of view. Right: The partial avatarization appears at the location of a printed marker and responds to user movements. if so, in which magnitudes. In this section, we grouped the knowledge on the impact of the avatarization's appearance and environmental factors. Little AR literature has covered other factors of influence yet.

\subsection{Impact of Appearance: The Uncanny Valley in AR}

The choice of the avatarization's appearance can be made over numerous characteristics, including the overall shape, body structure, size, fidelity or render style. Put together, these features produce distinct degrees of avatar anthropomorphism, realism, and resemblance with the user, contributing to different embodiment experiences [9]. The relationship between the avatar's appearance and the SoE was little studied in AR. VR research teaches us that their link (still largely debated) can be remarkably complex: while body ownership tends to be higher for realistic human models [130], [131], inhuman appearances (e.g. robot, abstract) will sometimes provide a stronger SoE than generic human avatars [7], [132] and sometimes not [133]. We are not aware of equivalent research in AR contexts and it seems unclear whether one appearance should be preferred over another for strong ownership illusions in AR.

Lugrin et al. [132] hypothesize that the culprit behind the inextricable interactions between $\mathrm{SoE}$ and appearance is the Uncanny Valley phenomenon, conceptualized by Mori et al. [134]. According to the theory, users feel a greater connection to anthropomorphic avatars as their degree of human resemblance rises until it reaches a point where the connection is brought to a sudden decline. This connection is reestablished to a greater extent from the point where the user considers the avatar to be realistic enough and would explain why human avatars produce a highly variable SoE.

In his study, Park [135] wondered if this phenomenon was expressed in the same way in AR. After evaluating the reactions of 50 participants to 7 (not embodied) avatars with varying human likeness, the author observed results unexpectedly contrasting with the ones of non-AR platforms: subjects actually found avatar models with the least human likeness to be the most uncanny, as opposed to those with the most human likeness, considered pleasant even with limited realism. Park suspects this to be linked to the dissonance in user expectations when observing real and virtual content being mixed. That is, inhuman and unrealistic avatars tend to create more inconsistency with the physical environment, causing the Uncanny Valley to appear at a low-end of human likeness level.

These results and their implications on the SoE remain to be more profoundly investigated. At this point, it is not clear whether experimenters seeking strong embodiment illusions should privilege realistic appearances to avoid falling into the Uncanny Valley. Gilbers et al. [1] seem to go along with this idea: upon reflecting on their AR VHI illusion results, they also theorized that Uncanny Valley effects could be responsible for the weak body ownership they observed. Rosa et al. [32] replicated this experiment and tried replacing the hand model with a projection of the participant's real hand, yielding positive subjective results this time (see Figure 7, bottom right). Unlike Park [135], they hypothesize that AR might have a similar but more sensitive response to Uncanny Valley effects as in VR and 
suggest future experiments to make exact copies of participants' hands. Such realistic rendering is attainable notably with 3D scans, high definition 3D renderings and video reconstructions.

However, these results contrast with a more recent experiment also conducted by Rosa et al. [32]. Using a headmounted VST display and VM stimulation over androgynous generic human and abstract block-shaped avatars, they found the SoE in AR to be modulated by the synchrony of the VM stimulation, but not by the appearance of the avatar. Although the avatar displayed limited realism in both conditions (no skin texture, no clothes), one could have expected a higher sense of ownership for the anthropomorphic avatar. These results echo those of Schwind et al. [7] in VR where androgynous human and abstract hands had similar effects on the user's perception of the avatar. If future research finds the Uncanny Valley to be negatively correlated to the SoE, these findings would then be opposed to the theory of Park [135].

Although experimental results are contrasting, research in $A R, V R$, and reality seems to hint that the perception of avatar appearance has a common basis across different embodiment media. However, the extent of this similarity remains to be clarified and many questions prevail on the appearance to choose to favor the SoE in AR.

\subsection{Impact of Body Structure Modifications in AR}

To which extent the avatar should match the human body schema for users to feel embodiment is another puzzling question. In VR, research has shown that appropriate stimulation can lead users as far as accepting a sixth finger [136] or even a tail added to their virtual body [137]. Rosa et al. [113] investigated whether this could also be feasible in AR with the user's real body. Their experiment let participants embody a virtual third arm with a VST HMD, stimulated in a VT and/or VM way. Their SoE measures showed that ownership, agency, and self-location could be evoked on the virtual arm while maintaining the real arms visible. The questionnaire answers also showed that the SoE was strongly experienced and that the simultaneous visibility of all three arms created the impression of having an arm at an additional location. Again, their findings reflect the capability of users to feel ownership and control towards differently structured avatars and extend what was previously observed in VR [137], [138].

In another AR publication, Feuchtner and Müller [57] questioned the extent of the modifications that can be made to the user's body structure before breaking the SoE. Similar to VR research [139], they managed to show that humans are capable of using arms extending up to more than twice their normal size (Figure 3, right) while still identifying them as their own. These results further illustrate the flexibility of our notion of body identity, but they also testify that there are limits to it: if the SoE was present for arms twice as long, it was considerably reduced for arms four times longer.

Lastly, it was repeatedly shown that the way we perceive our body is often on the fringe of reality, just as our recorded voice seems different from the one we hear when speaking [140], [141]. Such subjective distortion was witnessed in AR by Nimcharoen et al. [59] who showed that participants tended to identify with bodies slightly thinner than their own when presented several realistically deformed point clouds of themselves (see Figure 8). Nevertheless, not letting the choice of the avatar's appearance remains justified in the case of many applications such as in studies on the Proteus Effect where specific user behaviors are sought to be induced through avatar embodiment [142], [143], [144], [145].
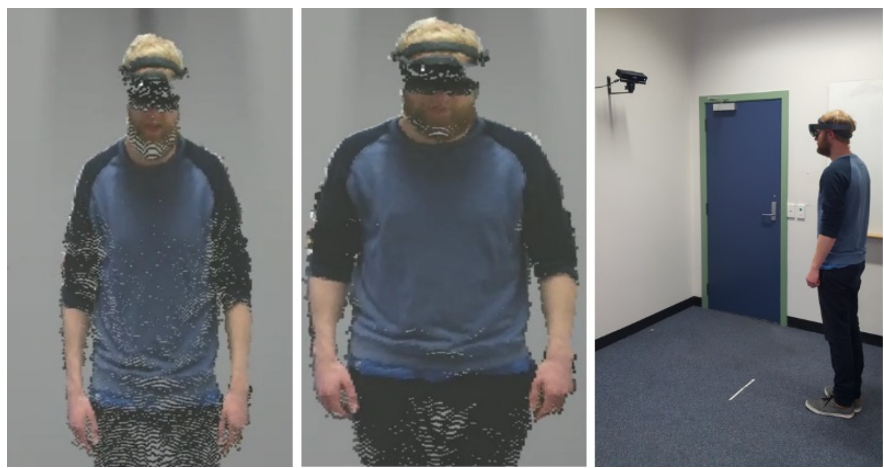

Fig. 8. Example of body morphology modifications applied on 3D point cloud reconstruction of a user in AR during full avatarization, extracted from [59]. Left: Thin body size. Center: Large body size. Right: Experimenter's point of view.

\subsection{Impact of Real Body Visibility}

Another point that should be noted is that, unlike in immersive VR, the real body of users usually remains visible during embodiment in AR. This is particularly true in OST or projection-based systems as they do not have the possibility to apply in-painting techniques like in VST. The transparency of their renderings constrain their users to hide real body parts by physical means, which can be inconvenient when done in 1PP. Wang et al. [58] attempted to clarify how much of a handicap this transparency can be by measuring the strength of VHIs displayed with different virtual:real light intensity ratios in $1 \mathrm{PP}$ AR (i.e. 0:1, 0.25:0.75, $0.5: 0.5,0.75: 0.25$, and 1:0). Their results show that ratios of 0.75:0.25 and 1:0 did not produce significantly different senses of ownership and agency towards the virtual hands. The authors conclude that they are equally "appropriate for promoting the virtual hand as part of the body".

Therefore, it seems that partial transparency is not a major impediment of VHIs as long as virtual hands remain more visible than the user's real hands. This result is encouraging for future research in OST as it implies that real body visibility is not a necessarily a limitation during $1 \mathrm{PP}$ embodiment experiences.

\subsection{Impact of Environmental Settings}

Whereas avatar-related factors were intensively studied in SoE research, very few papers have looked at whether the environmental context could play a part in body ownership illusions. VR and AR provide quite distinct visual feedback: one displays perfectly coherent virtual graphics while the other combines real world vision and 3D rendering. Therefore, it seems legitimate to question if the contrast between 
human vision and synthetic graphics could have an impact on how users experience self-avatarization.

Škola and Liarokapis [20] looked at the differences between the traditional RHI and the VHI in both VR and AR. Their findings show that AR and VR produce similar illusions, but in a weaker manner than when using a genuine rubber hand. IJsselsteijn et al. [146] previously examined the same illusion in mediated and unmediated studies. In their experiment, they replaced the rubber hand with a fake hand projected onto a tabletop. They found that applying VT stimulation with a real brush onto the projection caused a visual conflict between the real and projected material. This conflict impeded the embodiment of the virtual hand, regarded as physically incoherent.

In another study, Wolf et al. [21] compared user full avatarization perception in head-mounted VR and AR with a VST display. Subjects saw a photorealistic generic avatar through an interactive virtual mirror that offered VM feedback. The physical world viewed in the VST condition was faithfully reproduced in the VR condition. Unlike what the authors were expecting, participants did not report substantially different senses of ownership and presence in AR and in VR. They speculate that the immersion provided by their AR and VR systems was maybe too similar: the VST video resolution and VR graphics might have been too comparable for users to experience a difference. Unmediated vision of the AR condition with OST could have changed these results.

Genay et al. [22] investigated whether the mixing of real and virtual content can impact the SoE during $1 \mathrm{PP}$ partial avatarization in OST AR. In their experiment, participants controlled virtual robot hands in three AR environments: one where participants faced real objects only, one where they faced virtual objects only, and one where virtual and real objects were mixed together (Figure 9). They hypothesized that the presence of virtual objects in the environment would evoke a stronger SoE as it might help users to merge the virtual avatar with the real world, making it visually less contrasting with the rest of the scene.

Their results show that the condition with the mixed objects evoked a significantly stronger sense of ownership, but their secondary evaluation could not confirm this difference. Nevertheless, user feedback did show a general preference for environments mixing real and virtual objects during embodiment. They additionally comment on the highly varying sensitivity of users to visual contrast between the real and virtual objects. Some participants reported being disturbed by the mix of objects whereas others enjoyed it or did not pay attention to it. This seems to indicate that there is a degree of subjectivity in the perception of mixed environments.

We found no experiment comparing the SoE between OST and VST embodiment systems. Additionally, we did not find studies that evaluated the ownership illusions during interaction with mixed environments. Perhaps manipulating real objects with a virtual body could result in a different perception of the avatarization, but also of the affordances in a given environment: does embodying a larger avatar make users underestimate their ability to enter (real) narrow space? Further research will be needed to clarify these points.

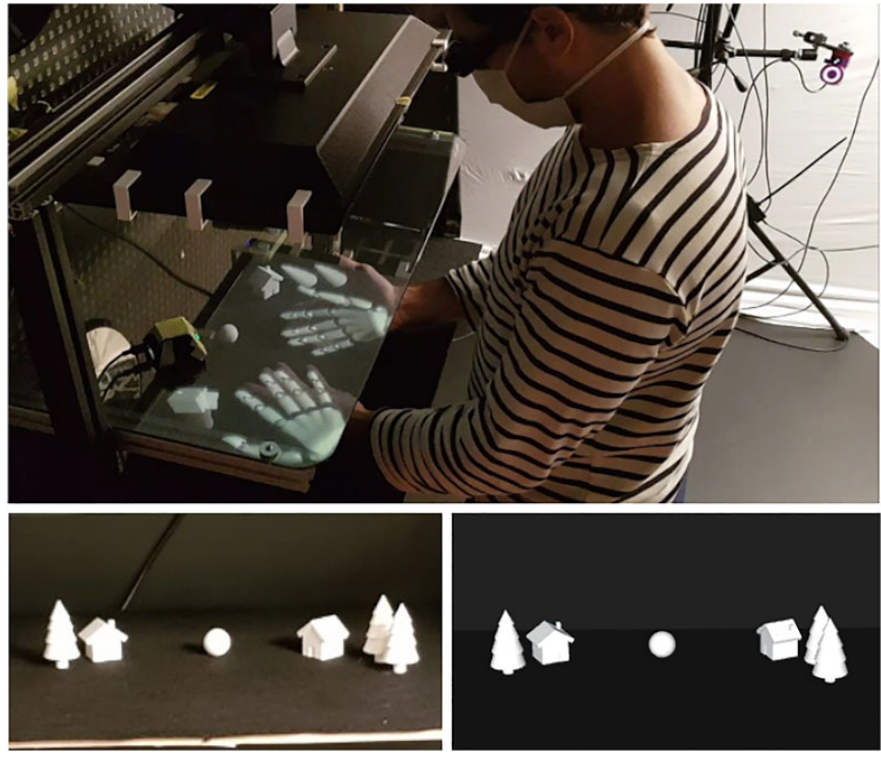

Fig. 9. Experimental setup of Genay et al. [22] studying the influence of virtual and real object presence during AR partial avatarization. Top: condition with mixed real and virtual objects. Bottom left: condition with only real objects. Bottom right: condition with only virtual objects.

\subsection{Other Important Cues in AR}

An important factor of the experiments mentioned previously is the timing of the applied stimuli. Zoulias et al. [147] emphasized the sensitivity of the illusion to this factor by showing that small delays have negative effects, especially when tactile stimulation precedes visual signal. This influence of synchrony was found in the AR version of the VHI elaborated by Gilbers [1], but its importance has not been assessed as deeply as in VR. For instance, Maselli and Slater [130] have shown that sufficiently deep immersion in VR can maintain a strong SoE in the presence of asynchronous stimuli; in that case, the user (wrongly) assumes the tactile sensations as coming from virtual objects in the scene. In AR, the possibility that the illusion could also subsist requires further investigation.

To intensify the senses of body ownership and selflocation, several methods other than synchronous VT and VM stimulation exist. Suzuki et al. [127] showed in their study that multisensory integration of exteroceptive and interoceptive signals (e.g. breathing activity) can modulate body ownership during AR VHIs. When making the virtual hand blush synchronously with the user's pulse, participants reported a stronger sense of ownership than with asynchronous cardiac feedback. However, ownership could occur in both conditions of synchrony when the participants were given the possibility to control the hand. The authors suspect movement signals to dominate the influence of the interoceptive signals and suggest that the participants' a priori willingness to accept the virtual hand might have played a role during the observation of asynchronous interoceptive feedback. While VR research seems to indicate that personality traits [10], psychological conditioning [148], and sensory suggestibility [11] are determinant of ownership illusions, the dominance of such top-down factors has not yet been discussed in AR to our knowledge. 


\section{Discussion}

The previous sections described experiments where the SoE could be evoked with different levels of body avatarization [32], [113], embodiment perspectives [31], [57], environments [21], [22], [59], display types [1], [12], [59], avatar appearances [59], [76], real body visibility [58], and sensory stimulation [20], [31], [127]. We conclude that, to some extent, the SoE can be elicited independently of the technological context as long as the appropriate stimuli and induction conditions are met.

That being said, it does not appear clear to us that equivalent real, virtual and hybrid environments will generate embodiment illusions of the same strengths, nor that the SoE will be affected by the same factors in the same ways. We discuss such possibilities in light of the knowledge collected throughout this review.

\subsection{The Sense of Embodying Avatars Across Realities}

Invariability of the SoE across contexts would be of utmost interest for applications that emphasize a personalized user experience. This includes video games, movies, and other arts [13], [149], but also medical therapies, rehabilitation and treatment of patients through partial and full avatarization (see Section 2).

While the systematic comparison made by Škola and Liarokapis [20] led them to conclude that AR, VR, and real-world VT stimulation induce similar embodiment experiences, their results also show some (small but real) differences between them. In particular, subjective results revealed a significant difference in the sense of ownership of a hand between the AR and real-world conditions, but not between AR and VR. Unlike Wolf et al. [21] who questioned their results (see Section 6.4), they did not discuss these differences and it is not clear whether the results would have been the same with an OST device.

Taking a step back, it seems likely that mediated and unmediated visualizations should logically provide different perceptions of virtual content, and therefore of AR avatarization. This is supported by well-documented perceptive biases such as depth underestimation that were found to vary between real-world, AR, and VR settings. Gaffary et al. [150] even showed that the "stiffness" of objects could also be experienced differently across display technologies: after pressing two pistons of identical stiffness in VST and VR, $60 \%$ of participants reported that the piston felt stiffer in VR than in VST AR. Their results go along with the theory that different biases could affect our senses across displays, including proprioception.

To conclude, it seems that the existing research comparing embodiment illusions across realities is too sparse to answer our inquiries. The small compound of literature on this subject (i.e. two publications [20], [21]) seems to indicate that equal SoEs can be generated across display modalities. Nevertheless, as AR environments can take extremely various shapes and be rendered in different ways, the impact of mixing realities will have to be further investigated. Unlike in the real world or in VR where visuals are homogeneous, current AR systems undergo a graphic contrast that allows discriminating virtual content [22]. Because the SoE relies on the coherence of visual and proprioceptive signals [5], it seems probable that AR will lead to different perceptions of body avatarization, varying with the system's capabilities.

\subsection{The Impact of Embodiment Illusions on Users in AR}

The influence of avatarization on user behavior, opinion, and cognition was not investigated in AR to our knowledge, and it cannot be assumed that Proteus Effects [17] also occur in AR without further investigation.

One paper that comes close to this subject in AR is the one of Jung et al. [151], involving on-person SAR projection. Their work investigated how anatomical information is perceived when projected directly onto the user's body, in comparison with on a plastic mannequin and on a screen. By displaying virtual lungs damaged by smoking, their experiment showed that a stronger negative impression was felt for projections directly set onto the location of their lungs. Hoang et al. [61] and Johnson and Sun [12] also proposed SAR systems for exploring psychological effects of on-person anatomical displays (muscles, blood vessels, etc.). Although their results were not evaluated in terms of SoE, they demonstrated a stronger level of "connectedness" and ownership with the projected content than with other projection supports, especially when the data was coherent with the participant's physiological state (e.g. synchronized heartbeat).

Supposing virtual embodiment does have effects on users in AR, Eckhoff et al. [18] hypothesize that realistic AR experiences could induce an even stronger virtual synesthesia than in virtual environments: being applied in a world that is familiar and known to be "real", illusions would be perceived more easily as actually being happening. As a consequence, they also argue that embodiment in AR could produce the same changes in behavior that were observed in VR.

This possibility raises many thought-provoking questions about the long-term usage of avatars in AR. Can the Proteus Effect alter our behaviors beyond the scope of VR experiments? Would this alteration be durable? How can we control and use it? What risks arise out of it? For the time being, these questions remain unanswered and the discussion related above is based on suppositions. Understanding these matters has immediate and tangible consequences for science, design, or production. As research has made it clear that virtual embodiment is useful for more than just decoding cognitive processes, we expect that future research will find great interest in clarifying these questions.

\subsection{Future Directions: Trends and Gaps of AR Embodi- ment Research}

Previous sections outlined existing gaps on the following topics: the influence of the avatar's appearance in AR, the existence AR-specific factors of influence, differences between OST, VST, VR and real world embodiment, and the impact of embodiment on user experience in AR. Through Table 1, we attempt to identify other under-explored research areas as well as trends in AR avatarization applications and SoE research. This table covers research as early as 2007 but over half of the presented papers were published from 2017, indicating a growing interest in AR avatarization. 
TABLE 1

Publications that put in place virtual body avatarization in AR, ordered by author name. POV stands for Point of View, $\mathrm{HCl}$ for Human-Computer Interaction, VHI for virtual hand illusion, and BOI for Body Ownership Illusion.

\begin{tabular}{|c|c|c|c|c|c|c|c|}
\hline Reference & $\begin{array}{l}\text { Continuum } \\
\text { Position }\end{array}$ & $\begin{array}{l}\text { Avatar } \\
\text { Appearance }\end{array}$ & Stimulus & POV & Display & Contribution & Application \\
\hline $\begin{array}{l}\text { Anderegg et al. [60] } \\
\text { (2018) }\end{array}$ & Full & $\begin{array}{l}\text { Abstract neutral } \\
\text { human model }\end{array}$ & $\mathrm{VM}$ & $3 P P$ & $\begin{array}{l}\text { VST with } \\
\text { smartphone }\end{array}$ & $\begin{array}{l}\text { Mobile phone } \\
\text { AR interaction }\end{array}$ & Video games \\
\hline Baskar [51] (2017) & Partial & $\begin{array}{l}\text { Simple neutral } \\
\text { human arm }\end{array}$ & $\mathrm{VM}$ & $1 \mathrm{PP}$ & OST HMD & $\begin{array}{l}\text { Prosthesis control } \\
\text { and simulation }\end{array}$ & Medical field \\
\hline $\begin{array}{l}\text { van Bommel [31] } \\
\text { (2017) }\end{array}$ & Full & $\begin{array}{l}\text { Abstract neutral } \\
\text { body }\end{array}$ & $\mathrm{VM}$ & $3 P P$ & VST HMD & $\begin{array}{l}\text { BOI, out-of-body } \\
\text { experiences }\end{array}$ & SoE research \\
\hline $\begin{array}{l}\text { Eckhoff et al. [18] } \\
\text { (2019) }\end{array}$ & $\begin{array}{l}\text { Partial, Ac- } \\
\text { cessorization }\end{array}$ & $\begin{array}{l}\text { Miscellaneous } \\
\text { augmentations }\end{array}$ & $\mathrm{VM}$ & $1 \mathrm{PP}$ & $\begin{array}{l}\text { OST, VST } \\
\text { SAR }\end{array}$ & $\begin{array}{l}\text { AR perception } \\
\text { and cognition }\end{array}$ & $\begin{array}{l}\text { Psychology, } \\
\text { medical field }\end{array}$ \\
\hline $\begin{array}{l}\text { Eisert et al. [42] } \\
\text { (2007) }\end{array}$ & $\begin{array}{l}\text { Accessoriza- } \\
\text { tion }\end{array}$ & Sports shoes & $\mathrm{VM}$ & $\begin{array}{l}\text { 3PP } \\
\text { (mirror) }\end{array}$ & Digital screen & Virtual mirror & Fashion \\
\hline $\begin{array}{l}\text { Feuchtner and } \\
\text { Müller [57] (2017) }\end{array}$ & Partial & $\begin{array}{l}\text { Realistic male } \\
\text { human arm }\end{array}$ & $\mathrm{VM}$ & $1 \mathrm{PP}$ & VST HMD & $\begin{array}{l}\text { Extensible arm } \\
\text { interaction }\end{array}$ & $\begin{array}{l}\text { SoE research, } \\
\text { HCI, implants }\end{array}$ \\
\hline $\begin{array}{l}\text { Genay et al. } \\
\text { [22] (2021) }\end{array}$ & Partial & $\begin{array}{l}\text { Realistic } \\
\text { robot hands }\end{array}$ & $\mathrm{VM}$ & $1 \mathrm{PP}$ & Fishtank & VHI & SoE research \\
\hline Gilbers [1] (2017) & Partial & $\begin{array}{l}\text { Simple neutral } \\
\text { human hand }\end{array}$ & VT, VM & $1 \mathrm{PP}$ & OST HMD & $\begin{array}{l}\text { VHI, supernume- } \\
\text { rary hand illusion }\end{array}$ & SoE research \\
\hline $\begin{array}{l}\text { Hamanishi et al. } \\
\text { [152] (2019) }\end{array}$ & Full & $\begin{array}{l}\text { Abstract neutral } \\
\text { body }\end{array}$ & $\mathrm{VM}$ & $3 \mathrm{PP}$ & OST HMD & $\begin{array}{l}\text { Improving posture } \\
\text { awareness }\end{array}$ & $\begin{array}{l}\text { Sports learning, } \\
\text { training }\end{array}$ \\
\hline $\begin{array}{l}\text { Heydrich et al. [153] } \\
\text { (2013) }\end{array}$ & Full & Video of user & VT & $3 \mathrm{PP}$ & VST HMD & $\begin{array}{l}\text { Out-of-body } \\
\text { experiences }\end{array}$ & SoE research \\
\hline $\begin{array}{l}\text { Hilsmann et al. [45] } \\
\text { (2009) }\end{array}$ & $\begin{array}{l}\text { Accesso- } \\
\text { rization }\end{array}$ & T-shirts & $\mathrm{VM}$ & $\begin{array}{l}\text { 3PP } \\
\text { (mirror) }\end{array}$ & Digital screen & $\begin{array}{l}\text { Retexturing of } \\
\text { clothes }\end{array}$ & Fashion \\
\hline $\begin{array}{l}\text { Hoang et al. [61] } \\
\text { (2018) }\end{array}$ & Full, partial & $\begin{array}{l}\text { Anatomical } \\
\text { user data }\end{array}$ & $\mathrm{VM}$ & $1 \mathrm{PP}$ & SAR & $\begin{array}{l}\text { Information } \\
\text { interfaces }\end{array}$ & $\begin{array}{l}\text { Education, } \\
\text { visualization }\end{array}$ \\
\hline $\begin{array}{l}\text { Javornik et al. } \\
\text { [29] (2017) }\end{array}$ & $\begin{array}{l}\text { Accessoriza- } \\
\text { tion }\end{array}$ & Facial make-up & $\mathrm{VM}$ & $\begin{array}{l}\text { 3PP } \\
\text { (mirror) }\end{array}$ & Digital screen & $\begin{array}{l}\text { Live facial } \\
\text { augmentation }\end{array}$ & $\begin{array}{l}\text { Exhibitions, } \\
\text { make-up }\end{array}$ \\
\hline $\begin{array}{l}\text { Johnson and Sun [12] } \\
\text { (2013) }\end{array}$ & Full, partial & $\begin{array}{l}\text { Anatomical } \\
\text { user data }\end{array}$ & $\mathrm{VM}$ & $1 \mathrm{PP}$ & SAR & $\begin{array}{l}\text { On-person } \\
\text { interfaces }\end{array}$ & $\begin{array}{l}\text { Education, } \\
\text { health }\end{array}$ \\
\hline $\begin{array}{l}\text { Kaneko et al. [56] } \\
(2019)\end{array}$ & Partial & Video of user & $\mathrm{VM}$ & $1 \mathrm{PP}$ & Digital screen & Stroke rehab. & Medical field \\
\hline $\begin{array}{l}\text { Kleinberger and } \\
\text { Panjwani [44] (2018) }\end{array}$ & $\begin{array}{l}\text { Partial, Ac- } \\
\text { cessorization }\end{array}$ & Dress & $\begin{array}{l}\text { VM, heart } \\
\text { rate, SCR }\end{array}$ & $1 \mathrm{PP}$ & SAR & $\begin{array}{l}\text { Wearable } \\
\text { computing }\end{array}$ & $\begin{array}{l}\text { Fashion, self- } \\
\text { expression }\end{array}$ \\
\hline $\begin{array}{l}\text { Lamounier Jr. et al. } \\
\text { [15] (2012) }\end{array}$ & Partial & $\begin{array}{l}\text { Simplified } \\
\text { prosthetic arm }\end{array}$ & $\mathrm{VM}$ & $1 \mathrm{PP}$ & VST HMD & $\begin{array}{l}\text { Prosthesis control } \\
\text { and simulation }\end{array}$ & Medical field \\
\hline $\begin{array}{l}\text { Martinez et al. [154] } \\
\text { (2014) }\end{array}$ & $\begin{array}{l}\text { Partial, Ac- } \\
\text { cessorization }\end{array}$ & Miscellaneous & $\mathrm{VM}$ & $\begin{array}{l}3 \mathrm{PP} \\
\text { (mirror) }\end{array}$ & $\begin{array}{l}\text { Optical com- } \\
\text { bining screen }\end{array}$ & Novel displays & $\begin{array}{l}\text { User safety, } \\
\text { exhibitions }\end{array}$ \\
\hline $\begin{array}{l}\text { Mercier-Ganady et } \\
\text { al. [155] (2014) }\end{array}$ & Partial & Brain activity & $\mathrm{VM}$ & $\begin{array}{l}\text { 3PP } \\
\text { (mirror) }\end{array}$ & $\begin{array}{l}\text { Optical com- } \\
\text { bining screen }\end{array}$ & $\begin{array}{l}\text { Information } \\
\text { interfaces }\end{array}$ & $\begin{array}{l}\text { Medical field, } \\
\text { BCI }\end{array}$ \\
\hline $\begin{array}{l}\text { Nimcharoen et al. } \\
\text { [59] (2018) }\end{array}$ & Full & $\begin{array}{l}\text { Video point } \\
\text { cloud of user }\end{array}$ & $\mathrm{VM}$ & $\begin{array}{l}\text { 3PP } \\
\text { (mirror) }\end{array}$ & OST HMD & BOI & $\begin{array}{l}\text { SoE research, } \\
\text { body perception }\end{array}$ \\
\hline $\begin{array}{l}\text { Nishino et al. [52] } \\
\text { (2017) }\end{array}$ & Partial & $\begin{array}{l}\text { Simplified } \\
\text { prosthetic arm }\end{array}$ & $\mathrm{VM}$ & $1 \mathrm{PP}$ & VST HMD & $\begin{array}{l}\text { Prosthesis control } \\
\text { and simulation }\end{array}$ & Medical field \\
\hline $\begin{array}{l}\text { Qian et al. [68] } \\
\text { (2019) }\end{array}$ & Partial & $\begin{array}{l}\text { Abstract neutral } \\
\text { hand }\end{array}$ & VT, VM & $1 \mathrm{PP}$ & $\begin{array}{l}\text { VST with } \\
\text { smartphone }\end{array}$ & $\begin{array}{l}\text { Mobile phone } \\
\text { AR interaction }\end{array}$ & Mobile apps \\
\hline $\begin{array}{l}\text { Treepong et al. [49] } \\
\text { (2018) }\end{array}$ & $\begin{array}{l}\text { Accessoriza- } \\
\text { tion }\end{array}$ & Make-up & $\mathrm{VM}$ & $\begin{array}{l}\text { 3PP } \\
\text { (mirror) }\end{array}$ & Digital screen & $\begin{array}{l}\text { Live facial } \\
\text { augmentation }\end{array}$ & Art, make-up \\
\hline $\begin{array}{l}\text { Rosa et al. [113] } \\
(2019)\end{array}$ & Partial & $\begin{array}{l}\text { Video point } \\
\text { cloud of user }\end{array}$ & VT, VM & 1PP & VST HMD & $\begin{array}{l}\text { Supernumerary } \\
\text { hand illusion }\end{array}$ & SoE research \\
\hline $\begin{array}{l}\text { Rosa et al. [32] } \\
(2019)\end{array}$ & Full & $\begin{array}{l}\text { Abstract neutral } \\
\text { body }\end{array}$ & $\mathrm{VM}$ & $3 P P$ & VST HMD & $\begin{array}{l}\text { BOI, out-of- } \\
\text { body experiences }\end{array}$ & SoE research \\
\hline $\begin{array}{l}\text { Saakes et al. [43] } \\
\text { (2016) }\end{array}$ & $\begin{array}{l}\text { Accessoriza- } \\
\text { tion }\end{array}$ & T-shirts & $\mathrm{VM}$ & $\begin{array}{l}\text { 1PP, 3PP } \\
\text { (mirror) }\end{array}$ & $\begin{array}{l}\text { SAR, Digital } \\
\text { screen }\end{array}$ & Virtual mirror & Fashion, art \\
\hline $\begin{array}{l}\text { Shibuya [79] } \\
\text { (2018) }\end{array}$ & Partial & $\begin{array}{l}\text { Realistic neutral } \\
\text { human hand }\end{array}$ & $\mathrm{VM}$ & $1 \mathrm{PP}$ & Fishtank & VHI & SoE research \\
\hline $\begin{array}{l}\text { Škola and Liarokapis } \\
\text { [20] (2016) }\end{array}$ & Partial & $\begin{array}{l}\text { Realistic neutral } \\
\text { human hand }\end{array}$ & VT & $1 \mathrm{PP}$ & VST HMD & RHI, VHI & SoE research \\
\hline $\begin{array}{l}\text { Slater et al. [74] } \\
(2008)\end{array}$ & Partial & $\begin{array}{l}\text { Realistic neutral } \\
\text { human arm }\end{array}$ & $\mathrm{VT}$ & $1 \mathrm{PP}$ & CAVE & VHI & SoE research \\
\hline $\begin{array}{l}\text { Suzuki et al. [127] } \\
\text { (2013) }\end{array}$ & Partial & $\begin{array}{l}\text { Video point } \\
\text { cloud of user }\end{array}$ & $\begin{array}{l}\text { VT, VM, } \\
\text { heart pulse }\end{array}$ & $1 \mathrm{PP}$ & VST HMD & VHI & SoE research \\
\hline $\begin{array}{l}\text { Wang et al. [156] } \\
\text { (2017) }\end{array}$ & Partial & $\begin{array}{l}\text { Realistic male } \\
\text { human hand }\end{array}$ & $\mathrm{VM}$ & $1 \mathrm{PP}$ & Fishtank & $\begin{array}{l}\text { Help typing } \\
\text { with hand tremor }\end{array}$ & Medical field \\
\hline $\begin{array}{l}\text { Watts et al. [75] } \\
\text { (2017) }\end{array}$ & Partial & $\begin{array}{l}\text { Anatomical } \\
\text { user data }\end{array}$ & $\mathrm{VM}$ & 1PP, 3PP & SAR & Novel displays & $\begin{array}{l}\text { Medical field, } \\
\text { education }\end{array}$ \\
\hline Wolf et al. [21] (2020) & Full & $\begin{array}{l}\text { Realistic } \\
\text { female body }\end{array}$ & $\mathrm{VM}$ & 3PP & VST HMD & BOI & $\begin{array}{l}\text { SoE research, } \\
\text { body perception }\end{array}$ \\
\hline
\end{tabular}




\subsubsection{Exploration of the Body Avatarization Continuum}

At first glance, it seems that partial avatarization was the most explored part of the body avatarization continuum. It was frequently used to embody virtual prosthetics [15], [52] or hands in studies of VHIs and variants, leaving out other human limbs [1], [20], [57], [127]. Body accessorization was the second most explored area of the continuum and was mainly used in application-oriented AR publications interested in fashion and innovative interaction [154]. Full avatarization was the least implemented. It was mostly used in SoE fundamental research (e.g. virtual body perception [1], [21], [31], [59]) and educational applications (e.g. medical visualization [12], [61]). Other application areas and psychological aspects of full-body ownership illusions remain relatively under-explored.

Research needs to be extended on the implementation and limitations of first-person embodiment in AR. Indeed, we found no example of 1PP views of full avatarization and rare examples in other parts of the continuum. As mentioned in Section 3.5, technical challenges are numerous when building such experience. Improving technical knowledge on 1PP AR avatarization would facilitate the construction of novel AR studies on how we perceive selfavatars replacing our real bodies. Therefore, implementation efforts to address avatar overlay issues would be welcome for future AR research on virtual embodiment.

\subsubsection{Multisensory AR Embodiment}

Another interesting lead is the creation of multisensory environments that not only involve the realistic integration of avatars but also touching sensations when embodying them. The skin forms the basis of our physical interactions with the world and allows us to define a mechanical understanding of our environment. By integrating active tactile feedback, virtual content becomes tangible and truly integrated into the real world, as physical objects [157]. Such feedback was moreover demonstrated as being a powerful tool to inspire SoE in both VR and AR research.

Yet, attempts to incorporate tactile sensations into AR applications are still in their infancy, as denoted by the rarity of VT stimulation in Table 1. According to Meli et al. [158], this is probably due to the slow diffusion of haptic interfaces adapted to AR that are user-friendly, lightweight, and affordable. Rosa [13] also outlines the under-exploration of other cues such as audio feedback in AR experiences. For instance, integrating heavy footstep sounds when embodying a weighty avatar could improve the credibility of the embodiment. More generally, visual and tactile active stimulation provide users with natural interaction feedback that can greatly enhance their understanding of the AR system's dynamics. Therefore, we encourage investigating further in this direction as it may participate in improving both embodiment illusions and general user experience.

\subsubsection{Promises of AR Avatars in the Medical Field}

AR provides one clear advantage over VR in medical applications: it allows patients to stay in environments they know. Not having to be immersed in a virtual environment allows incorporating therapies into the everyday lives of patients. Not breaking the normal course of a day might lead to higher acceptability of such treatments [18]. This could be particularly useful for the development of new strategies in a variety of medical contexts. Section 2.2 includes examples for prosthesis simulation and training, pain treatment, tremor stabilization, and stroke rehabilitation through AR partial avatarization (see Table 1). We expect research to continue trending for these particular applications, but also to expand to other types of medical processes and to other types of avatarization.

For example, future progress in brain-computer interfaces (BCI) or other methods (e.g. foot interfaces [159]) could lead AR avatarization to assist physically disabled people on a daily basis. It could notably improve the autonomy of quadriplegic people by letting them control virtual arms and interact with connected devices, as done in the study of Feuchtner and Muller [57].

Virtual avatarization also finds utmost interest in psychotherapies and assistance of mentally impaired people (e.g. autism spectrum disorders [160]). Treatment strategies for anorexia nervosa were repeatedly researched in VR while it is only beginning to be investigated in AR [21], [161], [162]. Currently, the number of AR studies is still insufficient to properly compare the efficiency of AR avatarization-based treatments with those in VR. More research is needed to clarify which medium is the most appropriate to treat each disorder.

\subsubsection{Learning from AR Embodiment Experiences}

Another interesting lead for AR embodiment research resides in education. Within AR, avatarization was often explored to improve user posture awareness and spatial perception by providing a 3PP visualization. For example, Hamanishi et al. [152] implemented an OST system providing an out-of-body viewpoint of the user rendered as an avatar animated in real time with motion capture. Soltani et al. [163] provided a complete survey of similar types of applications, but it seems that AR examples of full-bodied live avatarization are rare.

The possibility of a Proteus Effect in AR could open additional doors to novel educational methods for awarenessraising and skill enhancement. Indeed, Banakou et al. [142] showed that embodying a stereotype for superior intelligence like Albert Einstein in VR could increase executive functioning, problem-solving skills, and planning abilities. It seems the Proteus Effect may also impact artistic and motor abilities: embodiment of a dark-skinned casually dressed avatar by light-skinned people was shown to result in increased movement patterns while playing the drums than with a light-skinned formally dressed avatar [164]. One can easily imagine the advantages of exploiting these gains within specific real-world contexts by selecting the right full avatarization.

Alternatively, the Proteus Effect could be used to increase awareness and reduce bias. VR studies have found that participants would experience a reduction of implicit biases after embodying avatars with different gender [165], race or ethnicity [144], [166]. AR could provide a way to spend one's normal routine while under the skin of a different person and perhaps experience a longer-lasting effect than previously witnessed in VR. In this case, repeated or long 
exposures to the Proteus Effect through AR may serve to durably acquire skills and learning through avatarization.

\subsubsection{Role-playing and AR Gaming}

AR avatarization has an unequivocal potential in innovative story-telling and role-playing games (RPG). Through AR, users could instantly step into the shoes of a story's main character without having to immerse themselves in a virtual world. Taking such gaming experiences outside of VR allows seeing the physical world and exploiting its configuration. This brings supplementary opportunities for appealing game-plays involving interaction with real world objects (e.g. hiding behind real walls from attackers) and people [167], [168]. Additionally, AR visualizations are not concerned with many of the challenges linked to VR gaming, including avoiding real-world obstacles (e.g. chairs), dealing with cybersickness or navigating in large spaces with room restrictions. Currently, most AR games are handheld device applications that use screen interactions, leaving body avatarization aside [169]. Although mobile $\mathrm{AR}$ is an easy entry point, we believe HMDs will allow achieving the true potential of AR gaming by letting users embody game characters and customize their appearance. Given the fast-paced growth of AR technologies, we expect AR HMD games will soon include body avatarization [170].

\subsubsection{AR Avatars as Vehicles of the Self}

It is hard not to notice the large number of fashion-related applications in Table 1. In comparison with traditional product presentations, AR try-on systems were found to receive more favorable evaluations from users with low bodyrelated self-esteem [41]. These results seem to undergird the possibility of a much broader use of AR accessorization than virtual fittings for product consumption. In particular, body avatarization could serve to improve body satisfaction for those who have low self-image evaluations, but also to explore and express one's identity.

Entry-level applications (e.g. Snapchat ${ }^{9}$, Messenger ${ }^{10}$ ) have already made AR partial avatarization mainstream and it does not seem long before this extends to full avatarization. We believe the spread of AR avatarization applications will eventually constitute another step towards achieving what Harrell and Lim [171] described as the "Avatar Dream". This dream is the "culturally shared vision of a future in which, through computers, people can become whoever or whatever we want to be". According to Harrell, achieving this vision would allow people to gain empowerment when exploring and constructing their self-image, but also help them to negotiate the social constraints that limit who they can be in the real world. Online VR communities built on these principles are already starting to grow, but they are inherently limited to virtual environments. This opportunity for self-expression and social interaction could be extended seamlessly to the real world through AR.

With the advent of virtual influencers and similar entities, it seems that modern-day social media consumers and companies are already heading in this direction. Some users design social identities from scratch and materialize them as

9. https://www.snapchat.com/

10. https://www.messenger.com/ hyper-realistic avatars for which they create online profiles or video channels. Rapidly gaining in popularity, some of these profiles gather over 3 million followers and sometimes present videos of flesh-and-blood people interacting with the featured avatar (e.g. 19-year-old LilMiquela ${ }^{11}$ ). The decrease in the cost of tracking solutions and AR devices will eventually lead to more avatar-people live interactions. Regular users will then be able to embody their personalized self-avatars and convey the image they choose to be and to see of themselves.

As this may become possible in a near future, it seems urgent to better assess perceptual and cognitive characteristics of the embodiment of such avatars, but also to evaluate the medical, social and ethical risks of letting people take ownership of appearances that are not their own. Indeed, achieving the Avatar Dream might turn out harmful if it reinforces current social biases or tampers with human wellbeing, property and privacy rights. Future studies should strive in making this dream a positive one, fostering selffulfillment and encouraging face-to-face interaction instead of degrading human relations.

\section{CONCLUSION}

This paper provided an overview of previous research on the embodiment of virtual avatars in AR. We introduced a body avatarization continuum providing a taxonomy of user virtual representation, presented as a linear expansion of embodiment experiences. A guide of technical aspects related to ownership illusions was also proposed to help understand the key development possibilities and challenges of AR embodiment. We then covered the methods that exist to measure the SoE in AR and presented current knowledge on the factors of influence of the SoE in AR. Our analysis of past AR research on the SoE suggests that mixed environments are well capable of hosting body ownership illusions comparable to VR ones. However, factors of influence specific to AR environments were not thoroughly investigated and future research may reveal differences in the perception of AR embodiment. AR avatarization is a powerful tool for influencing human perception, physiology, and cognition in real-life situations beyond what VR currently offers. Understanding the dynamics underlying the SoE is essential to fully grasp how virtual avatarization can benefit us. At a time when AR technologies are being democratized faster than ever, extending AR embodiment studies will allow future applications to exploit ownership illusions outside of virtual environments. AR avatarization notably finds great potential in the medical field (e.g. prosthetics, rehabilitation, therapy), gaming industry, education, fashion, but also in social life and self-expression. We hope the discussion we provided will have raised questions and brought inspiration for future work to study the SoE in AR.

\section{REFERENCES}

[1] C. Gilbers, "The sense of embodiment in Augmented Reality: A third hand illusion," Master's thesis, Utrecht, Pays-Bas, 2017.

[2] K. Kilteni, R. Groten, and M. Slater, "The sense of embodiment in Virtual Reality," Presence: Teleoperators and Virtual Environments, vol. 21, pp. 373-387, Nov. 2012.

11. https://www.instagram.com/lilmiquela/ 
[3] M. Botvinick and J. Cohen, "Rubber hands 'feel' touch that eyes see," Nature, vol. 391, pp. 756-756, Feb. 1998.

[4] V. I. Petkova and H. H. Ehrsson, "If I were you: Perceptual illusion of body swapping," PLoS ONE, vol. 3, Dec. 2008.

[5] K. Kilteni, A. Maselli, K. P. Kording, and M. Slater, "Over my fake body: Body Ownership Illusions for studying the multisensory basis of own-body perception," Frontiers in Human Neuroscience, vol. 9, Mar. 2015.

[6] W. S. Bainbridge, Berkshire Encyclopedia of Human-computer Interaction. Great Barrington, Massachusetts, USA: Berkshire Publishing Group LLC, 2004.

[7] V. Schwind, P. Knierim, C. Tasci, P. Franczak, N. Haas, and N. Henze, "“These are not my hands!": Effect of gender on the perception of avatar hands in Virtual Reality," in Proceedings of the 2017 CHI Conference on Human Factors in Computing Systems. Denver, Colorado, USA: ACM Press, 2017, pp. 1577-1582.

[8] F. Argelaguet, L. Hoyet, M. Trico, and A. Lecuyer, "The role of interaction in virtual embodiment: Effects of the virtual hand representation," in 2016 IEEE Virtual Reality and 3D User Interfaces (VR), Greenville, South Carolina, USA, Mar. 2016, pp. 3-10.

[9] R. Fribourg, F. Argelaguet, A. Lécuyer, and L. Hoyet, “Avatar and sense of embodiment: Studying the relative preference between appearance, control and point of view," IEEE Transactions on Visualization and Computer Graphics, vol. 26, no. 5, pp. 2062-2072, 2020.

[10] D. Dewez, R. Fribourg, F. Argelaguet, L. Hoyet, D. Mestre, M. Slater, and A. Lécuyer, "Influence of personality traits and body awareness on the sense of embodiment in Virtual Reality," in 2019 IEEE International Symposium on Mixed and Augmented Reality (ISMAR). IEEE, 2019, pp. 123-134.

[11] A. Marotta, M. Tinazzi, C. Cavedini, M. Zampini, and M. Fiorio, "Individual differences in the rubber hand illusion are related to sensory suggestibility," PloS one, vol. 11, no. 12, p. e0168489, 2016.

[12] A. S. Johnson and Y. Sun, "Spatial Augmented Reality on person: Exploring the most personal medium," in International Conference on Virtual, Augmented and Mixed Reality. Springer, 2013, pp. 169174.

[13] N. Rosa, "Player/avatar body relations in multimodal Augmented Reality games," in Proceedings of the 18th ACM International Conference on Multimodal Interaction - ICMI 2016. Tokyo, Japan: ACM Press, 2016, pp. 550-553.

[14] S.-T. Noh, H.-S. Yeo, and W. Woo, "An HMD-based Mixed Reality system for avatar-mediated remote collaboration with bare-hand interaction," P. F. M. Imura and B. Mohler, Eds., Kyoto, Japan, 2015.

[15] E. Lamounier Jr, K. Lopes, A. Cardoso, and A. Soares, "Using Augmented Reality techniques to simulate myoelectric upper limb prostheses," J Bioengineer \& Biomedical Sci S, vol. 1, p. 2, 2012.

[16] L. Naert, C. Larboulette, and S. Gibet, "Lsf-animal: A motion capture corpus in french sign language designed for the animation of signing avatars," in Proceedings of The 12th Language Resources and Evaluation Conference, 2020, pp. 6008-6017.

[17] N. Yee and J. Bailenson, "The Proteus Effect: The effect of transformed self-representation on behavior," Human Communication Research, vol. 33, pp. 271-290, Jul. 2007.

[18] D. Eckhoff, A. Cassinelli, and C. Sandor, "Exploring perceptual and cognitive effects of extreme Augmented Reality experiences," Beijing, China, 2019, p. 4.

[19] G. Riva, R. M. Baños, C. Botella, F. Mantovani, and A. Gaggioli, "Transforming experience: the potential of augmented reality and virtual reality for enhancing personal and clinical change," Frontiers in psychiatry, vol. 7, p. 164, 2016.

[20] F. Škola and F. Liarokapis, "Examining the effect of body ownership in immersive Virtual and Augmented Reality environments," The Visual Computer, vol. 32, pp. 761-770, Jun. 2016.

[21] E. Wolf, N. Döllinger, D. Mal, C. Wienrich, M. Botsch, and M. E. Latoschik, "Body weight perception of females using photorealistic avatars in virtual and augmented reality," in 2020 IEEE International Symposium on Mixed and Augmented Reality (ISMAR). IEEE, 2020, pp. 462-473.

[22] A. Genay, A. Lécuyer, and M. Hachet, "Virtual, real or mixed: How surrounding objects influence the sense of embodiment in optical see-through experiences?" Frontiers in Virtual Reality, vol. 2, 2021.

[23] P. Milgram, H. Takemura, A. Utsumi, and F. Kishino, "Augmented Reality: A class of displays on the reality-virtuality continuum," in Telemanipulator and telepresence technologies, vol. 2351. International Society for Optics and Photonics, 1995, pp. 282-292.

[24] D. Kirsh, "Embodied cognition and the magical future of interaction design," ACM Transactions on Computer-Human Interaction (TOCHI), vol. 20, no. 1, pp. 1-30, 2013.

[25] P. Dourish, "Epilogue: Where the action was, wasn't, should have been, and might yet be," ACM Transactions on Computer-Human Interaction (TOCHI), vol. 20, no. 1, pp. 1-4, 2013.

[26] A. Shapiro, A. Leuski, and S. Marsella, "Ubebot: Voice-driven, personalized, avatar-based communicative video content in AR," in ACM SIGGRAPH 2019 Appy Hour on - SIGGRAPH '19. Los Angeles, California: ACM Press, 2019, pp. 1-2.

[27] R. T. Azuma, "A survey of Augmented Reality," Presence: Teleoperators and Virtual Environments, vol. 6, pp. 355-385, 1997.

[28] J. Frohner, G. Salvietti, P. Beckerle, and D. Prattichizzo, "Can wearable haptic devices foster the embodiment of virtual limbs?" IEEE Transactions on Haptics, vol. 12, pp. 339-349, Jul. 2019.

[29] A. Javornik, Y. Rogers, D. Gander, and A. Moutinho, "Magicface: Stepping into character through an Augmented Reality mirror," in Proceedings of the 2017 CHI Conference on Human Factors in Computing Systems, 2017, pp. 4838-4849.

[30] F. De Vignemont, "Embodiment, ownership and disownership," Consciousness and cognition, vol. 20, no. 1, pp. 82-93, 2011.

[31] J. van Bommel, "Presence and embodiment in Augmented Reality," Master's thesis, Utrecht, Pays-Bas, 2017.

[32] N. Rosa, J.-P. van Bommel, W. Hurst, T. Nijboer, R. C. Veltkamp, and P. Werkhoven, "Embodying an extra virtual body in Augmented Reality," in 2019 IEEE Conference on Virtual Reality and 3D User Interfaces (VR). Osaka, Japan: IEEE, Mar. 2019, pp. 1138 1139.

[33] B. Lenggenhager, T. Tadi, T. Metzinger, and O. Blanke, "Video ergo sum: manipulating bodily self-consciousness," Science, vol. 317, no. 5841, pp. 1096-1099, 2007.

[34] C. Harrison, H. Benko, and A. D. Wilson, "Omnitouch: wearable multitouch interaction everywhere," in Proceedings of the 24th annual ACM symposium on User interface software and technology, 2011, pp. 441-450.

[35] D. Parfit, "Personal identity," The Philosophical Review, vol. 80, no. 1, pp. 3-27, 1971.

[36] G. P. Stone, "Appearance and the self," Human behavior and social processes, pp. 86-118, 1962.

[37] H. Adam and A. D. Galinsky, "Enclothed cognition," Journal of experimental social psychology, vol. 48, no. 4, pp. 918-925, 2012.

[38] K. Johnson, S. J. Lennon, and N. Rudd, "Dress, body and self: Research in the social psychology of dress," Fashion and Textiles, vol. 1, no. 1, pp. 1-24, 2014.

[39] M. L. Slepian, S. N. Ferber, J. M. Gold, and A. M. Rutchick, "The cognitive consequences of formal clothing," Social Psychological and Personality Science, vol. 6, no. 6, pp. 661-668, 2015.

[40] M. W. Kraus and W. B. Mendes, "Sartorial symbols of social class elicit class-consistent behavioral and physiological responses: A dyadic approach." Journal of Experimental Psychology: General, vol. 143, no. 6, p. 2330, 2014.

[41] M. Y.-C. Yim and S.-Y. Park, "“I am not satisfied with my body, so I like augmented reality (AR)": Consumer responses to AR-based product presentations," Journal of Business Research, vol. 100, pp. 581-589, Jul. 2019. [Online]. Available: https://www.sciencedirect.com/science/article/pii/S0148296318305228

[42] P. Eisert, J. Rurainsky, and P. Fechteler, "Virtual mirror: Real-time tracking of shoes in Augmented Reality environments," in 2007 IEEE International Conference on Image Processing, vol. 2. IEEE, 2007, pp. II-557.

[43] D. Saakes, H.-S. Yeo, S.-T. Noh, G. Han, and W. Woo, "Mirror mirror: An on-body t-shirt design system," in Proceedings of the 2016 CHI Conference on Human Factors in Computing Systems, 2016, pp. 6058-6063.

[44] R. Kleinberger and A. Panjwani, "Digitally enchanted wear: a novel approach in the field of dresses as dynamic digital displays," in Proceedings of the Twelfth International Conference on Tangible, Embedded, and Embodied Interaction, 2018, pp. 27-34.

[45] A. Hilsmann and P. Eisert, "Tracking and retexturing cloth for real-time virtual clothing applications," in International Conference on Computer Vision/Computer Graphics Collaboration Techniques and Applications. Springer, 2009, pp. 94-105.

[46] S. G. Dacko, "Enabling smart retail settings via mobile augmented reality shopping apps," Technological Forecasting and 
Social Change, vol. 124, pp. 243-256, 2017. [Online]. Available: https://www.sciencedirect.com/science/article/pii/S0040162516304243

[47] P. Parekh, S. Patel, N. Patel, and M. Shah, "Systematic review and meta-analysis of augmented reality in medicine, retail, and games," Visual Computing for Industry, Biomedicine, and Art, vol. 3, no. 1, p. 21, Sep. 2020. [Online]. Available: https://doi.org/10.1186/s42492-020-00057-7

[48] A. H. Bermano, M. Billeter, D. Iwai, and A. Grundhöfer, “Makeup lamps: Live augmentation of human faces via projection," in Computer Graphics Forum, vol. 36, no. 2. Wiley Online Library, 2017, pp. 311-323.

[49] B. Treepong, H. Mitake, and S. Hasegawa, "Makeup Creativity Enhancement with an Augmented Reality Face Makeup System," Computers in Entertainment, vol. 16, no. 4, pp. 6:1-6:17, Nov. 2018. [Online]. Available: https://doi.org/10.1145/3277452

[50] A. Barbosa, E. A. Lamounier Junior, A. d. Oliveira Andrade, and A. Cardoso, "Virtual and Augmented Reality: A new approach to aid users of myoelectric prostheses," Oct. 2012.

[51] J. Baskar et al., "Holopham: An augmented reality training system for upper limb myoelectric prosthesis users," Ph.D. dissertation, Johns Hopkins University, 2017.

[52] W. Nishino, Y. Yamanoi, Y. Sakuma, and R. Kato, "Development of a myoelectric prosthesis simulator using Augmented Reality," in 2017 IEEE International Conference on Systems, Man, and Cybernetics (SMC). IEEE, 2017, pp. 1046-1051.

[53] S. Hoermann, E. A. Franz, and H. Regenbrecht, "Referred sensations elicited by video-mediated mirroring of hands," PLoS One, vol. 7, no. 12, p. e50942, 2012.

[54] C. Heinrich, M. Cook, T. Langlotz, and H. Regenbrecht, "My hands? importance of personalised virtual hands in a neurorehabilitation scenario," Virtual Reality, pp. 1-18, 2020.

[55] H. Regenbrecht, S. Hoermann, C. Ott, L. Mueller, and E. Franz, "Manipulating the experience of reality for rehabilitation applications," Proceedings of the IEEE, vol. 102, no. 2, pp. 170-184, 2014.

[56] F. Kaneko, K. Shindo, M. Yoneta, M. Okawada, K. Akaboshi, and M. Liu, "A case series clinical trial of a novel approach using augmented reality that inspires self-body cognition in patients with stroke: effects on motor function and resting-state brain functional connectivity," Frontiers in systems neuroscience, vol. 13, p. 76, 2019.

[57] T. Feuchtner and J. Müller, "Extending the body for interaction with reality," in Proceedings of the 2017 CHI Conference on Human Factors in Computing Systems. Denver, Colorado, USA: ACM Press, 2017, pp. 5145-5157.

[58] T.-Y. Wang, Y. Sato, M. Otsuki, H. Kuzuoka, and Y. Suzuki, "Effect of full body avatar in Augmented Reality remote collaboration," in 2019 IEEE Conference on Virtual Reality and 3D User Interfaces (VR). Osaka, Japan: IEEE, Mar. 2019, pp. 1221-1222.

[59] C. Nimcharoen, S. Zollmann, J. Collins, and H. Regenbrecht, "“Is That Me?" - Embodiment and body perception with an Augmented Reality mirror," in 2018 IEEE International Symposium on Mixed and Augmented Reality Adjunct (ISMAR-Adjunct). Munich, Germany: IEEE, Oct. 2018, pp. 158-163.

[60] R. Anderegg, L. Ciccone, and R. W. Sumner, "Puppetphone: puppeteering virtual characters using a smartphone," in Proceedings of the 11th Annual International Conference on Motion, Interaction, and Games, 2018, pp. 1-6.

[61] T. N. Hoang, H. S. Ferdous, F. Vetere, and M. Reinoso, "Body as a canvas: An exploration on the role of the body as display of digital information," in Proceedings of the 2018 Designing Interactive Systems Conference, 2018, pp. 253-263.

[62] H. G. Debarba, E. Molla, B. Herbelin, and R. Boulic, "Characterizing embodied interaction in first and third person perspective viewpoints," in 2015 IEEE Symposium on 3D User Interfaces (3DUI). IEEE, 2015, pp. 67-72.

[63] G. Gorisse, O. Christmann, E. A. Amato, and S. Richir, "First- and third-person perspectives in immersive virtual environments: Presence and performance analysis of embodied users," Frontiers in Robotics and AI, vol. 4, p. 33, Jul. 2017.

[64] M. Slater, B. Spanlang, M. V. Sanchez-Vives, and O. Blanke, "First person experience of body transfer in Virtual Reality," PLOS ONE, vol. 5, May 2010.

[65] V. I. Petkova, M. Khoshnevis, and H. H. Ehrsson, "The perspective matters! multisensory integration in ego-centric reference frames determines full-body ownership," Frontiers in psychology, vol. 2, p. 35, 2011.
[66] D. Medeiros, R. K. dos Anjos, D. Mendes, J. M. Pereira, A. Raposo, and J. Jorge, "Keep my head on my shoulders! why thirdperson is bad for navigation in VR," in Proceedings of the 24th ACM Symposium on Virtual Reality Software and Technology, 2018, pp. 1-10.

[67] "Impact of visuomotor feedback on the embodiment of virtual hands detached from the body," vol. 10. [Online]. Available: http://www.nature.com/articles/s41598-020-79255-5

[68] J. Qian, J. Ma, X. Li, B. Attal, H. Lai, J. Tompkin, J. F. Hughes, and J. Huang, "Portal-ble: Intuitive free-hand manipulation in unbounded smartphone-based Augmented Reality," in Proceedings of the 32nd Annual ACM Symposium on User Interface Software and Technology, 2019, pp. 133-145.

[69] D. Drascic and P. Milgram, "Perceptual issues in Augmented Reality," in Stereoscopic displays and Virtual Reality systems III, vol. 2653. International Society for Optics and Photonics, 1996, pp. 123-134.

[70] N. Toothman and M. Neff, "The impact of avatar tracking errors on user experience in VR," in 2019 IEEE Conference on Virtual Reality and 3D User Interfaces (VR). Osaka, Japan: IEEE, Mar. 2019, pp. 756-766.

[71] S. Zollmann, R. Grasset, T. Langlotz, W. H. Lo, S. Mori, and H. Regenbrecht, "Visualization Techniques in Augmented Reality: A Taxonomy, Methods and Patterns," IEEE Transactions on Visualization and Computer Graphics, pp. 1-1, 2020.

[72] E. Kruijff, J. E. Swan, and S. Feiner, "Perceptual issues in augmented reality revisited," in 2010 IEEE International Symposium on Mixed and Augmented Reality, Oct. 2010, pp. 3-12.

[73] J. P. Rolland and H. Fuchs, "Optical versus video see-through head-mounted displays in medical visualization," Presence: Teleoperators and Virtual Environments, vol. 9, pp. 287-309, Jun. 2000.

[74] M. Slater, "Towards a digital body: The virtual arm illusion," Frontiers in Human Neuroscience, vol. 2, 2008.

[75] I. Watts, P. Boulanger, and G. Kawchuk, "ProjectDR: Augmented Reality system for displaying medical images directly onto a patient," in Proceedings of the 23rd ACM Symposium on Virtual Reality Software and Technology, 2017, pp. 1-2.

[76] R. Gervais, J. Frey, A. Gay, F. Lotte, and M. Hachet, "Tobe: Tangible out-of-body experience," in Proceedings of the TEI'16: Tenth International Conference on Tangible, Embedded, and Embodied Interaction, 2016, pp. 227-235.

[77] M. Hachet, B. Bossavit, A. Cohé, and J.-B. de la Rivière, "Toucheo: multitouch and stereo combined in a seamless workspace," in Proceedings of the 24th annual ACM symposium on User interface software and technology, 2011, pp. 587-592.

[78] J. Mercier-Ganady, F. Lotte, E. Loup-Escande, M. Marchal, and A. Lécuyer, "The mind-mirror: See your brain in action in your head using eeg and Augmented Reality," in 2014 IEEE Virtual Reality (VR). IEEE, 2014, pp. 33-38.

[79] S. Shibuya, S. Unenaka, and Y. Ohki, "The relationship between the Virtual Hand Illusion and motor performance," Frontiers in Psychology, vol. 9, p. 2242, 2018.

[80] Z. Makhataeva and H. A. Varol, "Augmented reality for robotics: A review," Robotics, vol. 9, no. 2, p. 21, 2020.

[81] K. Ma and B. Hommel, "The role of agency for perceived ownership in the virtual hand illusion," Consciousness and cognition, vol. 36, pp. 277-288, 2015.

[82] A. Fernández-Baena, L. Salle, and D. Miralles, "Avatar: Tangible interaction and augmented reality in character animation," on Interacting with Smart Objects, p. 28, 2014.

[83] N. N. Griffin and E. Folmer, "Out-of-body locomotion: Vectionless navigation with a continuous avatar representation," in 25th ACM Symposium on Virtual Reality Software and Technology, 2019, pp. 1-8.

[84] M. Oshita, Y. Senju, and S. Morishige, "Character motion control interface with hand manipulation inspired by puppet mechanism," in Proceedings of the 12th ACM SIGGRAPH International Conference on Virtual-Reality Continuum and Its Applications in Industry, 2013, pp. 131-138.

[85] M. V. Sanchez-Vives, B. Spanlang, A. Frisoli, M. Bergamasco, and M. Slater, "Virtual hand illusion induced by visuomotor correlations," PloS one, vol. 5, no. 4, p. e10381, 2010.

[86] C. Farrer, M. Bouchereau, M. Jeannerod, and N. Franck, "Effect of distorted visual feedback on the sense of agency," Behavioural neurology, vol. 19, no. 1, 2, pp. 53-57, 2008.

[87] B. Spanlang, J.-M. Normand, D. Borland, K. Kilteni, E. Giannopoulos, A. Pomés, M. González-Franco, D. Perez-Marcos, 
J. Arroyo-Palacios, X. N. Muncunill et al., "How to build an embodiment lab: achieving body representation illusions in Virtual Reality," Frontiers in Robotics and AI, vol. 1, p. 9, 2014.

[88] Z. Cao, G. Hidalgo, T. Simon, S.-E. Wei, and Y. Sheikh, “Openpose: realtime multi-person $2 \mathrm{~d}$ pose estimation using part affinity fields," arXiv preprint arXiv:1812.08008, 2018.

[89] S. Kim, J. Kim, J.-H. Bae, and J. Park, "Real-time inverse kinematics technique for controlling redundant avatar arm," 062018.

[90] H.-S. Jang, J.-Y. Jeong, Y.-H. Kim, Y.-J. Yoon, and S.-J. Ko, “Augmented Reality with high frame rate for low computational power devices," in 2011 IEEE International Conference on Consumer Electronics-Berlin (ICCE-Berlin). IEEE, 2011, pp. 274-275.

[91] T. Waltemate, I. Senna, F. Hülsmann, M. Rohde, S. Kopp, M. Ernst, and M. Botsch, "The impact of latency on perceptual judgments and motor performance in closed-loop interaction in Virtual Reality," in Proceedings of the 22nd ACM Conference on Virtual Reality Software and Technology - VRST '16. Munich, Germany: ACM Press, 2016, pp. 27-35.

[92] A. U. Batmaz, M. R. Seraji, J. Kneifel, and W. Stuerzlinger, "No jitter please: Effects of rotational and positional jitter on $3 \mathrm{~d}$ midair interaction," in Proceedings of the Future Technologies Conference. Springer, 2020, pp. 792-808.

[93] F. Zheng, T. Whitted, A. Lastra, P. Lincoln, A. State, A. Maimone, and H. Fuchs, "Minimizing latency for Augmented Reality displays: Frames considered harmful," in 2014 IEEE International Symposium on Mixed and Augmented Reality (ISMAR). IEEE, 2014, pp. 195-200.

[94] R. Zabels, R. Smukulis, R. Fenuks, A. Kučiks, E. Linina, K. Osmanis, and I. Osmanis, "Reducing motion to photon latency in multi-focal augmented reality near-eye display," in Optical Architectures for Displays and Sensing in Augmented, Virtual, and Mixed Reality $(A R, V R, M R) I I$, vol. 11765. International Society for Optics and Photonics, 2021, p. 117650W.

[95] P. Lincoln, A. Blate, M. Singh, T. Whitted, A. State, A. Lastra, and H. Fuchs, "From motion to photons in 80 microseconds: Towards minimal latency for virtual and augmented reality," IEEE transactions on visualization and computer graphics, vol. 22, no. 4, pp. 1367-1376, 2016

[96] H. Kato and M. Billinghurst, "Marker tracking and hmd calibration for a video-based Augmented Reality conferencing system," in Proceedings 2nd IEEE and ACM International Workshop on Augmented Reality (IWAR'99). IEEE, 1999, pp. 85-94.

[97] S. Garrido-Jurado, R. Munoz-Salinas, F. J. Madrid-Cuevas, and R. Medina-Carnicer, "Generation of fiducial marker dictionaries using mixed integer linear programming," Pattern Recognition, vol. 51, pp. 481-491, 2016.

[98] F. J. Romero-Ramirez, R. Muñoz-Salinas, and R. Medina-Carnicer, "Speeded up detection of squared fiducial markers," Image and vision Computing, vol. 76, pp. 38-47, 2018.

[99] H. Durrant-Whyte and T. Bailey, "Simultaneous localization and mapping: part I," IEEE robotics \& automation magazine, vol. 13, no. 2, pp. 99-110, 2006.

[100] G. Reitmayr, T. Langlotz, D. Wagner, A. Mulloni, G. Schall, D. Schmalstieg, and Q. Pan, "Simultaneous localization and mapping for Augmented Reality," in 2010 International Symposium on Ubiquitous Virtual Reality. IEEE, 2010, pp. 5-8.

[101] J. Collins, H. Regenbrecht, and T. Langlotz, "Visual coherence in mixed reality: A systematic enquiry," Presence: Teleoperators and Virtual Environments, vol. 26, no. 1, pp. 16-41, 2017.

[102] M. Akçayır and G. Akçayır, "Advantages and challenges associated with augmented reality for education: A systematic review of the literature," Educational Research Review, vol. 20, pp. 1-11, 2017.

[103] T. Masood and J. Egger, "Augmented reality in support of industry 4.0-implementation challenges and success factors," Robotics and Computer-Integrated Manufacturing, vol. 58, pp. 181-195, 2019.

[104] R. Likert, "A technique for the measurement of attitudes," Archives of psychology, vol. 22, 1932.

[105] M. Gonzalez-Franco and T. C. Peck, "Avatar embodiment. towards a standardized questionnaire," Frontiers in Robotics and AI, vol. 5, p. 74, Jun. 2018.

[106] D. Roth, J.-L. Lugrin, M. E. Latoschik, and S. Huber, "Alpha ivbo - construction of a scale to measure the illusion of virtual body ownership," in Proceedings of the 2017 CHI Conference Extended Abstracts on Human Factors in Computing Systems. Denver, Colorado, USA: ACM Press, 2017, pp. 2875-2883.
[107] M. R. Longo, F. Schüür, M. P. Kammers, M. Tsakiris, and P. Haggard, "What is embodiment? a psychometric approach," Cognition, vol. 107, pp. 978-998, Jun. 2008.

[108] M. Slater, "How colorful was your day? why questionnaires cannot assess presence in virtual environments," Presence: Teleoperators E Virtual Environments, vol. 13, no. 4, pp. 484-493, 2004.

[109] D. Roth and M. E. Latoschik, "Construction of the Virtual Embodiment Questionnaire (VEQ)," IEEE Transactions on Visualization and Computer Graphics, vol. 26, no. 12, pp. 3546-3556, Dec. 2020.

[110] T. C. Peck and M. Gonzalez-Franco, "Avatar embodiment. a standardized questionnaire," Frontiers in Virtual Reality, vol. 1, p. $44,2021$.

[111] M. Rohde, M. Di Luca, and M. O. Ernst, "The Rubber Hand Illusion: feeling of ownership and proprioceptive drift do not go hand in hand," PloS one, vol. 6, no. 6, p. e21659, 2011.

[112] H. Holle, N. McLatchie, S. Maurer, and J. Ward, "Proprioceptive drift without illusions of ownership for rotated hands in the "Rubber Hand Illusion" paradigm," Cognitive neuroscience, vol. 2, no. 3-4, pp. 171-178, 2011.

[113] N. Rosa, R. C. Veltkamp, W. Hürst, T. Nijboer, C. Gilbers, and P. Werkhoven, "The supernumerary hand illusion in Augmented Reality," ACM Transactions on Applied Perception, vol. 16, pp. 1-20, Aug. 2019.

[114] G. L. Moseley, N. Olthof, A. Venema, S. Don, M. Wijers, A. Gallace, and C. Spence, "Psychologically induced cooling of a specific body part caused by the illusory ownership of an artificial counterpart," Proceedings of the National Academy of Sciences, vol. 105, no. 35, pp. 13169-13173, 2008.

[115] M. Slater, D. Pérez Marcos, H. Ehrsson, and M. V. Sanchez-Vives, "Towards a digital body: the virtual arm illusion," Frontiers in human neuroscience, vol. 2, p. 6, 2008.

[116] H.-G. Jo, M. Wittmann, T. Hinterberger, and S. Schmidt, "The readiness potential reflects intentional binding," Frontiers in human neuroscience, vol. 8, p. 421, 2014.

[117] J. W. Moore and S. S. Obhi, "Intentional binding and the sense of agency: a review," Consciousness and cognition, vol. 21, no. 1, pp. 546-561, 2012.

[118] M. Seignette and M. S. Christensen, "Absence of electroencephalographic evidence of implicit action-effect intentional binding with varying effect probability," bioRxiv, pp. 2020-12, 2021.

[119] A. Schettler, V. Raja, and M. L. Anderson, "The embodiment of objects: Review, analysis, and future directions," Frontiers in Neuroscience, vol. 13, p. 1332, Dec. 2019.

[120] K. C. Armel and V. S. Ramachandran, "Projecting sensations to external objects: Evidence from skin conductance response," Proceedings of the Royal Society of London. Series B: Biological Sciences, vol. 270, pp. 1499-1506, Jul. 2003.

[121] S. Aglioti, N. Smania, M. Manfredi, and G. Berlucchi, "Disownership of left hand and objects related to it in a patient with right brain damage," Neuroreport, vol. 8, no. 1, pp. 293-296, 1996.

[122] L. Raz, P. L. Weiss, and M. Reiner, "The Virtual Hand Illusion and body ownership," in Haptics: Perception, Devices and Scenarios, M. Ferre, Ed. Heidelberg, Germany: Springer, 2008, pp. 367-372.

[123] M. Teräs, "You are your avatar is you: Phenomenological literature review of virtual embodiment in virtual environments," Tech. Rep., Jul. 2015.

[124] H. Ehrsson, "The concept of body ownership and its relation to multisensory integration," The New Handbook of Multisensory Processes, pp. 775-792, Jan. 2012.

[125] E. Kokkinara and M. Slater, "Measuring the effects through time of the influence of visuomotor and visuotactile synchronous stimulation on a virtual Body Ownership Illusion," Perception, vol. 43, pp. 43-58, Jan. 2014.

[126] M. Tsakiris, G. Prabhu, and P. Haggard, "Having a body versus moving your body: How agency structures body-ownership," Consciousness and Cognition, vol. 15, pp. 423-432, Jun. 2006.

[127] K. Suzuki, S. N. Garfinkel, H. D. Critchley, and A. K. Seth, "Multisensory integration across exteroceptive and interoceptive domains modulates self-experience in the rubber-hand illusion | Elsevier Enhanced Reader," Neuropsychologia, 2013.

[128] S. Jeon and S. Choi, "Haptic Augmented Reality: Taxonomy and an example of stiffness modulation," Presence: Teleoperators and Virtual Environments, vol. 18, no. 5, pp. 387-408, 2009.

[129] H. H. Ehrsson, N. P. Holmes, and R. E. Passingham, "Touching a rubber hand: Feeling of body ownership is associated with 
activity in multisensory brain areas," The Journal of Neuroscience, vol. 25, pp. 10564-10573, Nov. 2005.

[130] A. Maselli and M. Slater, "The building blocks of the Full Body Ownership Illusion," Frontiers in Human Neuroscience, vol. 7, Mar. 2013.

[131] T. Waltemate, D. Gall, D. Roth, M. Botsch, and M. E. Latoschik, "The impact of avatar personalization and immersion on virtual body ownership, presence, and emotional response," IEEE transactions on visualization and computer graphics, vol. 24, no. 4, pp. 1643-1652, 2018.

[132] J.-L. Lugrin, J. Latt, and M. E. Latoschik, “Avatar anthropomorphism and illusion of body ownership in VR," in 2015 IEEE Conference on Virtual Reality and 3D User Interfaces (VR). Arles, France: IEEE, Mar. 2015, pp. 229-230.

[133] L. Lin and S. Jörg, "Need a hand?: how appearance affects the virtual hand illusion," in Proceedings of the ACM Symposium on Applied Perception. Anaheim California: ACM, Jul. 2016, pp. 69-76. [Online]. Available: https://dl.acm.org/doi/10.1145/2931002.2931006

[134] M. Mori, K. F. MacDorman, and N. Kageki, "The uncanny valley [from the field]," IEEE Robotics Automation Magazine, vol. 19, pp. 98-100, Jun. 2012.

[135] S. J. Park, "Is Augmented Reality in denial of the convention? examining the presence of Uncanny Valley in Augmented Reality," no. TR2019-866, May 2019.

[136] L. Hoyet, F. Argelaguet, C. Nicole, and A. Lécuyer, "“'wow! I have six fingers!": Would you accept structural changes of your hand in VR?" Frontiers in Robotics and AI, vol. 3, May 2016.

[137] W. Steptoe, A. Steed, and M. Slater, "Human tails: ownership and control of extended humanoid avatars," IEEE transactions on visualization and computer graphics, vol. 19, no. 4, pp. 583-590, 2013.

[138] A. Guterstam, V. I. Petkova, and H. H. Ehrsson, "The illusion of owning a third arm," PloS one, vol. 6, no. 2, p. e17208, 2011.

[139] K. Kilteni, J.-M. Normand, M. V. Sanchez-Vives, and M. Slater, "Extending body space in immersive Virtual Reality: A very long arm illusion," PLoS ONE, vol. 7, p. e40867, Jul. 2012.

[140] I. V. Piryankova, H. Y. Wong, S. A. Linkenauger, C. Stinson, M. R. Longo, H. H. Bülthoff, and B. J. Mohler, "Owning an overweight or underweight body: Distinguishing the physical, experienced and virtual body," PLoS ONE, vol. 9, p. e103428, Aug. 2014.

[141] P. D. Slade and G. Russell, "Awareness of body dimensions in anorexia nervosa: Cross-sectional and longitudinal studies," Psychological medicine, vol. 3, no. 2, pp. 188-199, 1973.

[142] D. Banakou, S. Kishore, and M. Slater, "Virtually being einstein results in an improvement in cognitive task performance and a decrease in age bias," Frontiers in Psychology, vol. 9, p. 917, Jun. 2018.

[143] S. Buisine, J. Guegan, J. Barré, F. Segonds, and A. Aoussat, "Using avatars to tailor ideation process to innovation strategy," Cognition, Technology \& Work, vol. 18, no. 3, pp. 583-594, 2016.

[144] T. C. Peck, S. Seinfeld, S. M. Aglioti, and M. Slater, "Putting yourself in the skin of a black avatar reduces implicit racial bias," Consciousness and cognition, vol. 22, no. 3, pp. 779-787, 2013.

[145] J. Guegan, S. Buisine, F. Mantelet, N. Maranzana, and F. Segonds, "Avatar-mediated creativity: When embodying inventors makes engineers more creative," Computers in Human Behavior, vol. 61, pp. 165-175, 2016.

[146] W. A. IJsselsteijn, Y. A. W. de Kort, and A. Haans, "Is this my hand I see before me? the Rubber Hand Illusion in reality, Virtual Reality, and Mixed Reality," Presence: Teleoperators and Virtual Environments, vol. 15, no. 4, pp. 455-464, 2006.

[147] I. D. Zoulias, W. S. Harwin, Y. Hayashi, and S. J. Nasuto, "Milliseconds matter: Temporal order of visuo-tactile stimulation affects the ownership of a virtual hand," in 10th International Conference, EuroHaptics 2016, Proceedings, Part II, F. Bello, H. Kajimoto, and Y. Visell, Eds., vol. 9775, Haptics: Perception, Devices, Control, and Applications. London, UK: Springer International Publishing, 2016, pp. 479-489.

[148] E. Walsh, D. Guilmette, M. R. Longo, J. W. Moore, D. Oakley, P. Halligan, M. Mehta, and Q. Deeley, "Are you suggesting that's my hand? the relation between hypnotic suggestibility and the Rubber Hand Illusion," Perception, vol. 44, pp. 709-723, Jun. 2015.

[149] M. Billinghurst, H. Kato, and I. Poupyrev, "The magicbook moving seamlessly between reality and virtuality," IEEE Computer Graphics and Applications, vol. 21, pp. 6-9, Feb. 2001.
[150] Y. Gaffary, B. Le Gouis, M. Marchal, F. Argelaguet, B. Arnaldi, and A. Lécuyer, "Ar feels "softer" than vr: Haptic perception of stiffness in augmented versus virtual reality," IEEE transactions on visualization and computer graphics, vol. 23, no. 11, pp. 2372-2377, 2017.

[151] S. Jung, J. Lee, F. Biocca, and J. W. Kim, "Augmented Reality in the health domain: projecting spatial Augmented Reality visualizations on a perceiver's body for health communication effects," Cyberpsychology, Behavior, and Social Networking, vol. 22, no. 2, pp 142-150, 2019.

[152] N. Hamanishi, T. Miyaki, and J. Rekimoto, "Assisting viewpoint to understand own posture as an avatar in-situation," in Proceedings of the 5th International ACM In-Cooperation HCI and UX Conference, 2019, pp. 1-8.

[153] L. Heydrich, T. J. Dodds, J. E. Aspell, B. Herbelin, H. H. Bülthoff, B. J. Mohler, and O. Blanke, "Visual capture and the experience of having two bodies - evidence from two different Virtual Reality techniques," Frontiers in Psychology, vol. 4, 2013.

[154] D. Martinez Plasencia, F. Berthaut, A. Karnik, and S. Subramanian, "Through the combining glass," in Proceedings of the 27th annual ACM symposium on User interface software and technology, 2014, pp. 341-350.

[155] J. Mercier-Ganady, F. Lotte, E. Loup-Escande, M. Marchal, and A. Lécuyer, "The mind-mirror: See your brain in action in your head using EEG and Augmented Reality," in 2014 IEEE Virtual Reality (VR), 2014, pp. 33-38.

[156] K. Wang, D. Iwai, and K. Sato, "Supporting Trembling Hand Typing Using Optical See-Through Mixed Reality," IEEE Access, vol. 5, pp. 10700-10708, 2017.

[157] X. Yu, Z. Xie, Y. Yu, J. Lee, A. Vazquez-Guardado, H. Luan, J. Ruban, X. Ning, A. Akhtar, D. Li et al., "Skin-integrated wireless haptic interfaces for virtual and Augmented Reality," Nature, vol. 575, no. 7783, pp. 473-479, 2019.

[158] L. Meli, C. Pacchierotti, G. Salvietti, F. Chinello, M. Maisto, A. De Luca, and D. Prattichizzo, "Combining wearable finger haptics and Augmented Reality: User evaluation using an external camera and the microsoft hololens," IEEE Robotics and Automation Letters, vol. 3, no. 4, pp. 4297-4304, 2018.

[159] E. Abdi, E. Burdet, M. Bouri, and H. Bleuler, "Control of a Supernumerary Robotic Hand by Foot: An Experimental Study in Virtual Reality," PLOS ONE, vol. 10, no. 7, p. e0134501, Jul. 2015.

[160] R. O. Kellems, C. Charlton, K. S. Kversøy, and M. Győri, “Exploring the use of virtual characters (avatars), live animation, and augmented reality to teach social skills to individuals with autism," Multimodal Technologies and Interaction, vol. 4, no. 3, p. 48, 2020.

[161] S. C. Mölbert, A. Thaler, B. J. Mohler, S. Streuber, J. Romero, M. J. Black, S. Zipfel, H.-O. Karnath, and K. E. Giel, "Assessing body image in anorexia nervosa using biometric self-avatars in Virtual Reality: Attitudinal components rather than visual body size estimation are distorted," Psychological Medicine, vol. 48, pp. 642-653, Mar. 2018.

[162] J. Park, "The effect of virtual avatar experience on body image discrepancy, body satisfaction and weight regulation intention," Cyberpsychology: Journal of Psychosocial Research on Cyberspace, vol. 12 , no. 1, 2018.

[163] P. Soltani and A. H. Morice, "Augmented reality tools for sports education and training," Computers $\mathcal{E}$ Education, vol. 155, p. 103923, 2020.

[164] K. Kilteni, I. Bergstrom, and M. Slater, "Drumming in immersive virtual reality: the body shapes the way we play," IEEE transactions on visualization and computer graphics, vol. 19, no. 4, pp. 597-605, 2013.

[165] K. Beltran, C. Rowland, N. Hashemi, A. Nguyen, L. Harrison, S. Engle, and B. F. Yuksel, "Reducing implicit gender bias using a virtual workplace environment," in Extended Abstracts of the 2021 CHI Conference on Human Factors in Computing Systems, 2021, pp. $1-7$.

[166] D. Banakou, P. D. Hanumanthu, and M. Slater, "Virtual embodiment of white people in a black virtual body leads to a sustained reduction in their implicit racial bias," Frontiers in Human Neuroscience, vol. 10, Nov. 2016.

[167] B. H. Thomas, "A survey of visual, mixed, and augmented reality gaming," Computers in Entertainment (CIE), vol. 10, no. 1, pp. 133, 2012. 
[168] T. Nilsen, S. Linton, and J. Looser, "Motivations for augmented reality gaming," Proceedings of FUSE, vol. 4, pp. 86-93, 2004.

[169] D. Chatzopoulos, C. Bermejo, Z. Huang, and P. Hui, "Mobile augmented reality survey: From where we are to where we go," Ieee Access, vol. 5, pp. 6917-6950, 2017.

[170] Y. Xu, E. Barba, I. Radu, M. Gandy, R. Shemaka, B. Schrank, B. MacIntyre, and T. Tseng, "Pre-patterns for designing embodied interactions in handheld augmented reality games," in 2011 IEEE International Symposium on Mixed and Augmented Reality-Arts, Media, and Humanities. IEEE, 2011, pp. 19-28.

[171] D. F. Harrell and C.-U. Lim, "Reimagining the avatar dream: Modeling social identity in digital media," Communications of the ACM, vol. 60, no. 7, pp. 50-61, 2017.

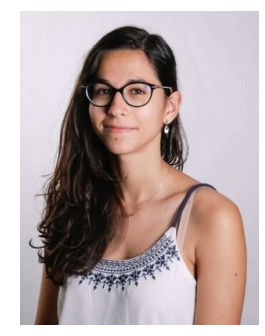

Adélaïde Genay Adélaïde Genay graduated in 2019 from the French national engineering school of Bordeaux ENSEIRB-MATMECA, receiving her computer science degree specialized in multimedia and video game technologies. She is now a Ph.D. student at Inria Bordeaux SudOuest, France, in the Potioc team. Her research interests include avatars, augmented reality, and virtual embodiment.

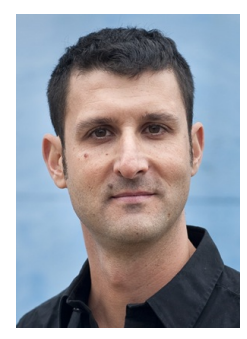

Anatole Lécuyer Anatole Lécuyer is a senior researcher and head of the Hybrid research team at Inria, the French National Institute for Research in Computer Science and Control, Rennes, France. His main research interests include virtual reality, 3D user interfaces, haptic interaction, and brain-computer interfaces. He currently is an associate editor of IEEE Transactions on Visualization and Computer Graphics, and of Frontiers in Virtual Environments and Presence journals. He was Program Chair of IEEE Virtual Reality Conference (2015-2016) and IEEE Symposium on 3D User Interfaces (2012-2013). He obtained the Inria-French Academy of Sciences Young Researcher Prize in 2013.

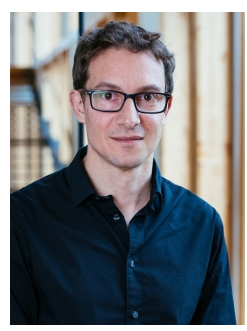

Martin Hachet Martin Hachet is a senior researcher at Inria Bordeaux where he leads the Potioc research team. His main research interest is in Human-Computer Interaction where he explores approches that are notably based on VR, $\mathrm{AR}, \mathrm{MR}$ and Tangible Interaction. He is particular interested in the domains of Education, Art, and Well-being. He was a Steering committee member of the IEEE Symposium on 3D User Interfaces (2011-2017) and Program chair of IHM (2017), 3DUI (2010-2011) and VRST (2008). 